S1

\title{
A General Strategy for Stereoselective Glycosylations
}

Jin-Hwan Kim, Hai Yang, Jin Park and Geert-Jan Boons*

Complex Carbohydrate Research Center, University of Georgia, 315 Riverbend Road, Athens, GA 30602.gjboons@,ccrc.uga.edu

\section{Supporting Information}

\section{Experimental Data for Compounds}

General Procedures. All reactions were carried out under a positive pressure of argon, unless otherwise noted. All chemicals were purchased from commercial suppliers and used without further purification, unless otherwise noted. Dichloromethane was distilled from calcium hydride under $\mathrm{N}_{2}$. Toluene was distilled under nitrogen from molten sodium. $N, N$-Dimethylformamide (DMF) was distilled under nitrogen from barium oxide. Column chromatography was performed on silica gel 60 (EM Science, 70-230 mesh). Reactions were monitored by TLC on Kieselgel $60 \mathrm{~F}_{254}$ (EM Science) and the compounds were detected by examination under UV light and visualized by dipping the plates in a cerium sulfate-ammonium molybdate solution followed by heating. Organic solutions were concentrated by rotary evaporation below $40{ }^{\circ} \mathrm{C}$ under reduced pressure. Molecular sieves ( $3 \AA$ and $4 \AA$ ), used for reactions, were crushed and activated in vacuo at $390{ }^{\circ} \mathrm{C}$ during $8 \mathrm{~h}$ in the first instance and then for $2-3 \mathrm{~h}$ at $390{ }^{\circ} \mathrm{C}$ directly prior to

application. Optical rotations were measured with a 'Jasco P-1020' polarimeter. ${ }^{1} \mathrm{H}$ NMR 
and ${ }^{13} \mathrm{C}$ NMR spectra were recorded with a Varian Inova 300 spectrometer and a Varian Inova 500 spectrometer equipped with Sun workstations. Chemical shifts are reported in parts per million (ppm) downfield from tetramethylsilane. Data are presented as follow: Chemical shift, multiplicity $(\mathrm{s}=$ singlet, $\mathrm{d}=$ doublet, $\mathrm{t}=$ triplet, $\mathrm{dd}=$ double of doublet, $\mathrm{m}$ $=$ multiplet and/or multiple resonances), integration, coupling constant in Hertz (Hz). High-resolution mass spectra were run in a JMS SX/SX102A tandem mass spectrometer, equipped with FAB source. The matrix used was DHB and the internal standards ultramark 1621 and PEG.

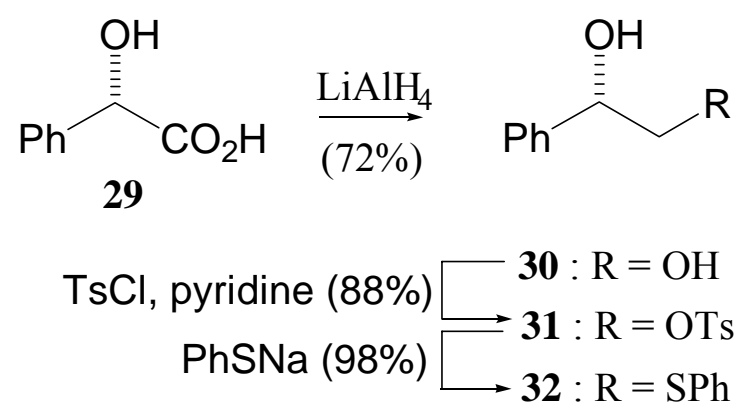

Scheme 1. Preparation of (S)-2-(phenylthiomethyl)benzyl acetate (33)

(S)-Phenyl-1,2-ethanediol (30). To a solution of (S)-mandelic acid (10.0 g, $65.7 \mathrm{mmol})$ in diethyl ether $(50 \mathrm{~mL})$ was added slowly $\mathrm{LiAlH}_{4}(5.0 \mathrm{~g}, 131 \mathrm{mmol})$. After the reaction mixture was refluxed for $2 \mathrm{~h}$, ethyl acetate $(20 \mathrm{~mL})$ was added slowly and then poured into ice water $(100 \mathrm{~mL})$. The organic phase was separated and dried $\left(\mathrm{MgSO}_{4}\right)$, filtered and the filtrate was concentrated in vacuo. The residue was purified by recrystallization (hexane/ethyl acetate) to afford $30(6.54 \mathrm{~g}, 72 \%)$ as a white solid, $R_{f}=0.24$ (ethyl acetate/hexane, $1 / 1) ;[\alpha]^{\mathrm{D}}{ }_{20}+60.5\left(c 1.15, \mathrm{CHCl}_{3}\right) ;{ }^{1} \mathrm{H}$ NMR $\left(500 \mathrm{MHz}, \mathrm{CDCl}_{3}\right) \delta 7.37-$ 
S3

$7.26(\mathrm{~m}, 5 \mathrm{H}, \mathrm{ArH}), 4.82(\mathrm{dd}, 1 \mathrm{H}, J=3.6,8.1 \mathrm{~Hz}, \mathrm{CHOH}), 3.76(\mathrm{dd}, 1 \mathrm{H}, J=3.6,11.4 \mathrm{~Hz}$, CHHOH), 3.66 (dd, $1 \mathrm{H}, J=8.1,11.4 \mathrm{~Hz}, \mathrm{CH} H \mathrm{OH}), 2.42$ (brs, 2H, OH).

(Ref : Bradshaw, Jerald S. Jolley, Scott t.; Izatt, Reed M. J. Org. Chem. 1982, 47, 12291232.)

(S)-1-Phenyl-2-(tosyloxy)ethanol (31). To a solution of $30(4.18 \mathrm{~g}, 30.3 \mathrm{mmol})$ in $\mathrm{CH}_{2} \mathrm{Cl}_{2}(60 \mathrm{~mL})$ were sequentially added $\mathrm{Bu}_{2} \mathrm{SnO}(151 \mathrm{mg}, 0.6 \mathrm{mmol}), \mathrm{TsCl}(5.77 \mathrm{~g}, 30.3$ mmol) and $\mathrm{Et}_{3} \mathrm{~N}(4.2 \mathrm{~mL}, 30.3 \mathrm{mmol})$. After stirring at room temperature for $3 \mathrm{~h}$, the reaction mixture was diluted with $\mathrm{CH}_{2} \mathrm{Cl}_{2}(60 \mathrm{~mL})$ and washed with brine $(40 \mathrm{~mL})$. The organic phase was dried $\left(\mathrm{MgSO}_{4}\right)$, filtered and the filtrate was concentrated in vacuo. The residue was purified by silica gel column chromatography ( $25 \%$ ethyl acetate in hexane) to afford $31(7.81 \mathrm{~g}, 88 \%)$ as a colorless syrup, $R_{f}=0.69$ (ethyl acetate/hexane, $\left.1 / 1\right)$; $[\alpha]^{\mathrm{D}}{ }_{20}+43.6\left(c 1.12, \mathrm{CHCl}_{3}\right) ;{ }^{1} \mathrm{H} \mathrm{NMR}\left(500 \mathrm{MHz}, \mathrm{CDCl}_{3}\right) \delta 7.70(\mathrm{~d}, 2 \mathrm{H}, J=8.4 \mathrm{~Hz}$, ArH), 7.37-7.26 (m, 7H, ArH), 5.01-4.96 (m, 1H, CHOH), 4.16 (dd, 1H, $J=3.3,10.5$ Hz, CHHOTs), 4.05 (dd, 1H, $J=8.4,10.5, \mathrm{~Hz}, \mathrm{CH} H \mathrm{OTs}), 2.53$ (d, 1H, $J=3.3 \mathrm{~Hz}$, $\mathrm{CHOH}), 2.45\left(\mathrm{~s}, 3 \mathrm{H}, \mathrm{CH}_{3}\right)$.

(Ref: Pandey, Rajesh Kumar; Fernandes, Rodney A.; Kumar, Pradeep. Tetrahedron Lett. 2002, 43, 4425-4426.)

(S)-1-Phenyl-2-(phenylthio)ethanol (32). To a solution of 31 (7.81 g, $26.7 \mathrm{mmol})$ in THF $(40 \mathrm{~mL})$ was added benzenethiol, sodium salt $(90 \%, 5.11 \mathrm{~g}, 35.0 \mathrm{mmol})$. The reaction mixture was stirred at room temperature overnight, then concentrated in vacuo. The residue was dissolved with dichloromethane $(50 \mathrm{~mL})$ and washed with brine $(50 \mathrm{~mL})$. 
The organic phase was dried $\left(\mathrm{MgSO}_{4}\right)$, filtered and the filtrate was concentrated in vacuo. The residue was purified by silica gel column chromatography (25\% ethyl acetate in hexane) to afford $32(6.0 \mathrm{~g}, 98 \%)$ as a colorless syrup, $R_{f}=0.30$ (ethyl acetate/hexane, $1 / 4) ;[\alpha]^{\mathrm{D}}{ }_{20}+21.0\left(c \quad 0.27, \mathrm{CHCl}_{3}\right) ;{ }^{1} \mathrm{H}$ NMR $\left(500 \mathrm{MHz}, \mathrm{CDCl}_{3}\right) \delta 7.34-7.12(\mathrm{~m}, 10 \mathrm{H}$, ArH), $4.73(\mathrm{dd}, 1 \mathrm{H}, J=2.1,5.7 \mathrm{~Hz}, \mathrm{CHOH}), 3.33(\mathrm{dd}, 1 \mathrm{H}, J=2.1,8.4 \mathrm{~Hz}, \mathrm{CHHSPh}$ ), 3.10 (dd, 1H, $J=5.7,8.4 \mathrm{~Hz}, \mathrm{CH} H \mathrm{SPh}), 2.81(\mathrm{~s}, 1 \mathrm{H}, \mathrm{OH})$.

(Ref: Christoffers, Jens; Roessler, Ulrich. Tetrahedron; Asymmetry. 1999, 10, 12071215.)

(S)-(Phenylthiomethyl)benzyl acetate (33). To a solution of $32(6.0 \mathrm{~g}, 26.0 \mathrm{mmol})$ in pyridine $(30 \mathrm{~mL})$ was added acetic anhydride $(5.0 \mathrm{~mL}, 52.0 \mathrm{mmol})$. After stirring at $0{ }^{\circ} \mathrm{C}$ for $2 \mathrm{~h}$, the reaction mixture was quenched with saturated sodium bicarbonate solution $(50 \mathrm{~mL})$ and then diluted with dichloromethane $(50 \mathrm{~mL})$. After separation, the organic phase was washed with saturated sodium bicarbonate solution $(50 \mathrm{~mL} \times 3)$ and brine $(50$ $\mathrm{mL})$. The organic phase was dried $\left(\mathrm{MgSO}_{4}\right)$, filtered and the filtrate was concentrated in vacuo. The residue was purified by silica gel column chromatography $(20 \%$ ethyl acetate in hexane) to afford $33(5.82 \mathrm{~g}, 82 \%)$ as a colorless syrup, $R_{f}=0.47$ (ethyl acetate/hexane, $1 / 4) ;[\alpha]_{20}^{\mathrm{D}}+31.6\left(c 1.52, \mathrm{CHCl}_{3}\right) ;{ }^{1} \mathrm{H}$ NMR $\left(500 \mathrm{MHz}, \mathrm{CDCl}_{3}\right) \delta 7.39-$ $7.20(\mathrm{~m}, \mathrm{ArH}), 5.89(\mathrm{dd}, 1 \mathrm{H}, J=5.4,8.1 \mathrm{~Hz}), 3.41(\mathrm{dd}, 1 \mathrm{H}, J=8.1,14.1 \mathrm{~Hz}, C H \mathrm{HSPh})$, 3.23 (dd, $1 \mathrm{H}, J=5.4,14.1 \mathrm{~Hz}, \mathrm{CH} H \mathrm{SPh}) 2.02\left(\mathrm{~s}, 3 \mathrm{H}, \mathrm{COCH}_{3}\right)$.

(Ref: Bortolini, O.; Furia, F.; Licini, G.; Modena, G. Phosphorus Sulfur. 1988, 37, 171174.) 


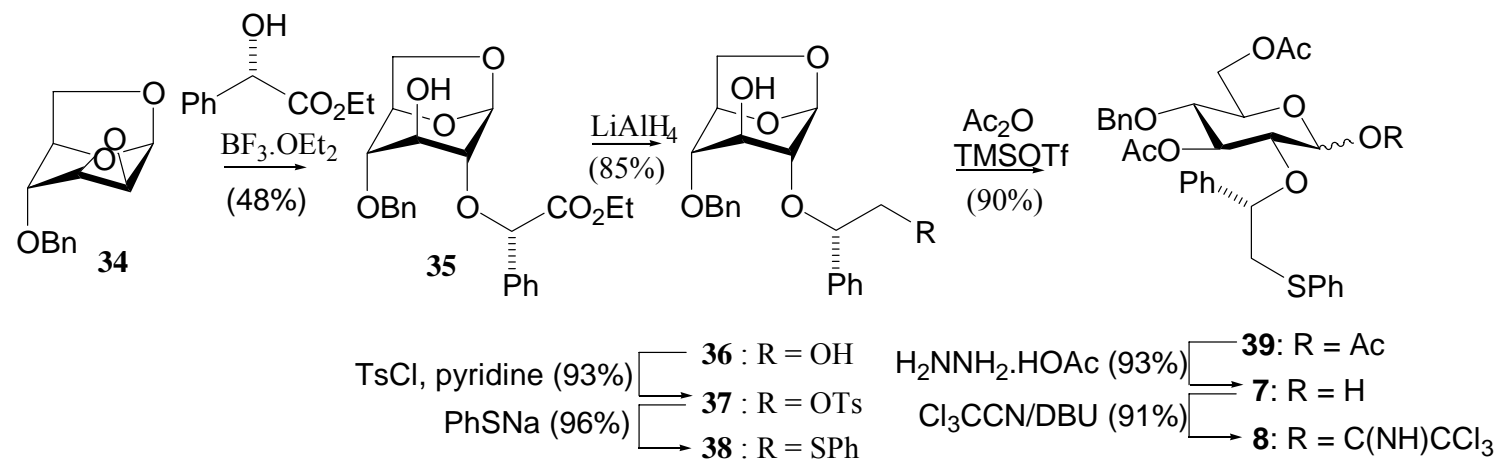

Scheme 2. Preparation of glucosyl donor 8

\section{1,6-Anhydro-4-O-benzyl-2-O-(S)-(ethoxycarbonyl)benzyl- $\beta$-D-glucopyranose}

(35). Boron trifluoride diethyl etherate ( $543 \mu \mathrm{L}, 4.27 \mathrm{mmol})$ was added dropwise to a solution of 1,6,2,3-dianhydro-4-O-benzyl- $\beta$-D-mannopyranose (34) (5 g, $21.3 \mathrm{mmol}$ ), (S)-ethyl mandelate $(11.5 \mathrm{~g}, 63.9 \mathrm{mmol})$ and activated molecular sieves $(4 \AA)$ in toluene $(20 \mathrm{~mL})$ at room temperature. After $1 \mathrm{~h}$, the reaction mixture was quenched with saturated aqueous $\mathrm{NaHCO}_{3}(30 \mathrm{~mL})$ and then diluted with ethyl acetate $(30 \mathrm{~mL})$. The organic phase was dried $\left(\mathrm{MgSO}_{4}\right)$, filtered and the filtrate was concentrated in vacuo. The residue was purified by silica gel column chromatography $(20 \%$ ethyl acetate in hexane) to afford 35 (4.23 g, 48\%): colorless syrup, $R_{f}=0.34$ (ethyl acetate/hexane, $1 / 1) ;[\alpha]^{20}{ }_{\mathrm{D}}=+40.3^{\circ}\left(c=1.0, \mathrm{CHCl}_{3}\right) ;{ }^{1} \mathrm{H}$ NMR $\left(300 \mathrm{MHz}, \mathrm{CDCl}_{3}\right) \delta$ 7.25-7.48 (m, 10H, aromatic), 5.54 (s, 1H, H-1), $5.22(\mathrm{~s}, 1 \mathrm{H},>\mathrm{CHPh}), 4.63$ (d, 1H, $J$ $=12.3 \mathrm{~Hz}, \mathrm{CHHPh}), 4.61(\mathrm{~d}, 1 \mathrm{H}, J=12.3 \mathrm{~Hz}, \mathrm{CHHPh}), 4.54(\mathrm{~d}, 1 \mathrm{H}, J=5.4 \mathrm{~Hz}, \mathrm{H}-5)$, 4.13-4.23 (m, 2H, $\left.\mathrm{COOCH}_{2} \mathrm{CH}_{3}\right), 3.88(\mathrm{t}, 1 \mathrm{H}, J=4.8 \mathrm{~Hz}, \mathrm{H}-3), 3.73(\mathrm{~d}, 1 \mathrm{H}, J=7.5$ Hz, H-6a), 3.61 (dd, 1H, J= 5.4, 7.2 Hz, H-6b), 3.33 (d, 1H, J=4.8, H-2), 3.29 (d, $1 \mathrm{H}, J=5.1, \mathrm{H}-4), 2.61(\mathrm{~b}, 1 \mathrm{H}, \mathrm{OH}), 1.21\left(\mathrm{t}, 3 \mathrm{H}, J=7.2 \mathrm{~Hz}, \mathrm{COOCH}_{2} \mathrm{CH}_{3}\right) ;{ }^{13} \mathrm{C} \mathrm{NMR}$ 
$\left(75 \mathrm{MHz}, \mathrm{CDCl}_{3}\right) \delta 171.07,137.79,136.01,128.73,128.59,128.43,127.78,127.75$, $127.50,101.80,80.49,80.17,80.02,75.48,71.80,71.25,66.85,61.43,14.01$; HR MALDI-TOF MS: m/z: calcd for $\mathrm{C}_{23} \mathrm{H}_{26} \mathrm{O}_{7}[\mathrm{M}+\mathrm{Na}]^{+}$: 437.1577 ; found: 437.1532 .

\section{1,6-Anhydro-4-O-benzyl-2-O-\{(S)-2-(hydroxymethyl)benzyl\}- $\beta$-D-glucopyranose}

(36). To a solution of $35(1.20 \mathrm{~g}, 2.90 \mathrm{mmol})$ in THF $(35 \mathrm{~mL})$ was added $\mathrm{LiAlH}_{4}(220$

$\mathrm{mg}, 5.8 \mathrm{mmol})$. After the reaction mixture was refluxed for $4 \mathrm{~h}$, diluted with ethyl acetate $(20 \mathrm{~mL})$ and then poured into ice water $(10 \mathrm{~mL})$. The organic phase was dried $\left(\mathrm{MgSO}_{4}\right)$, filtered and the filtrate was concentrated in vacuo. The residue was purified by silica gel column chromatography ( $67 \%$ ethyl acetate in hexane) to afford 36 ( 0.92 g, $85 \%$ ) as colorless syrup, $R_{f}=0.24$ (ethyl acetate/hexane, $\left.2 / 1\right) ;[\alpha]^{20}{ }_{\mathrm{D}}=+28.36^{\circ}(c$ $\left.=5.0, \mathrm{CHCl}_{3}\right) ;{ }^{1} \mathrm{H} \mathrm{NMR}\left(500 \mathrm{MHz}, \mathrm{CDCl}_{3}\right) \delta 7.35-7.27(\mathrm{~m}, 10 \mathrm{H}$, aromatic), $5.57(\mathrm{~s}$, 1H, H-1), 4.67 (d, 1H, $J=12.0 \mathrm{~Hz}, \mathrm{CHHPh}), 4.60-4.58$ (m, 1H, H-6a), 4.58 (d, 1H, $J$ $=12.0 \mathrm{~Hz}, \mathrm{CHHPh}), 4.50(\mathrm{~d}, 1 \mathrm{H}, J=5.0 \mathrm{~Hz}, \mathrm{H}-5), 3.79-3.77$ (m, 2H, H-7, H-3), 3.71 (dd, $1 \mathrm{H}, J=9.0,12.0 \mathrm{~Hz}, \mathrm{H}-8 \mathrm{a}$ ), 3.62 (dd, $1 \mathrm{H}, J=5.0,5.5 \mathrm{~Hz}, \mathrm{H}-4), 3.59$ (dd, $1 \mathrm{H}, J=$ 3.5, $12.0 \mathrm{~Hz}, \mathrm{H}-8 \mathrm{~b}), 3.25-3.24$ (m, 2H, H-2, H-6b); ${ }^{13} \mathrm{C}$ NMR (75 MHz, $\left.\mathrm{CDCl}_{3}\right) \delta$ $138.22,137.79,128.63,128.59,128.52,128.40,127.92,127.84,127.08,100.61$, 82.60, 79.36, 78.27, 75.40, 71.72, 70.73, 67.08, 66.38; HR MALDI-TOF MS: m/z: calcd for $\mathrm{C}_{21} \mathrm{H}_{24} \mathrm{O}_{6}[\mathrm{M}+\mathrm{Na}]^{+}$: 395.1471; found: 395.1405 .

\section{1,6-Anhydro-4-O-benzyl-2-O-\{(S)-2-(tosyloxymethyl)benzyl\}- $\beta$-D-glucopyranose}

(37). To a solution of $36(0.78 \mathrm{~g}, 2.09 \mathrm{mmol})$ in pyridine $(10 \mathrm{~mL})$ was added $\mathrm{TsCl}$ ( $0.44 \mathrm{~g}, 2.32 \mathrm{mmol})$. After stirring at room temperature for $2 \mathrm{~h}$, the reaction mixture 


\section{S7}

was diluted with $\mathrm{CH}_{2} \mathrm{Cl}_{2}(20 \mathrm{~mL})$ and washed with brine $(40 \mathrm{~mL})$. The organic phase was dried $\left(\mathrm{MgSO}_{4}\right)$, filtered and the filtrate was concentrated in vacuo. The residue was purified by silica gel column chromatography (33\% ethyl acetate in hexane) to afford $37(1.02 \mathrm{~g}, 93 \%)$ as colorless syrup, $R_{f}=0.38$ (ethyl acetate/hexane, 1/1); $[\alpha]^{20}{ }_{\mathrm{D}}=-13.11^{\circ}\left(c=4.5, \mathrm{CHCl}_{3}\right) ;{ }^{1} \mathrm{H} \mathrm{NMR}\left(500 \mathrm{MHz}, \mathrm{CDCl}_{3}\right) \delta 7.75-7.74(\mathrm{~m}, 2 \mathrm{H}$, aromatic), 7.34-7.28 (m, 12H, aromatic), $5.44(\mathrm{~s}, 1 \mathrm{H}, \mathrm{H}-1), 4.75(\mathrm{dd}, 1 \mathrm{H}, J=5.0,7.0$ $\mathrm{Hz}, \mathrm{H}-7), 4.58$ (d, 1H, $J=12.0 \mathrm{~Hz}, \mathrm{CHHPh}), 4.51$ (d, 1H, $J=12.0 \mathrm{~Hz}, \mathrm{CHHPh})$, 4.52-4.50 (m, 1H, H-4), 4.13-4.11 (m, 2H, H-8a,8b), 3.70-3.68 (d, 1H, J=7.0 Hz, H6a), 3.63-3.60 (m, 1H, H-3), 3.62 (d,1H, $J=7.0 \mathrm{~Hz}, \mathrm{H}-6 \mathrm{~b}), 3.23$ (d, 1H, $J=5.0 \mathrm{~Hz}$, $\mathrm{H}-2), 3.19(\mathrm{~d}, 1 \mathrm{H}, J=5.0 \mathrm{~Hz}, \mathrm{H}-5), 2.42\left(\mathrm{~s}, 3 \mathrm{H}, \mathrm{CH}_{3}\right) ;{ }^{13} \mathrm{C} \mathrm{NMR}\left(75 \mathrm{MHz}, \mathrm{CDCl}_{3}\right) \delta$ $144.89,137.79,136.94,132.85,129.88,128.78,128.77,128.48,127.93,127.86$, $127.78,127.01,101.31,80.85,80.20,79.71,75.50,72.76,71.73,70.97,66.78,21.63$; HR MALDI-TOF MS: m/z: calcd for $\mathrm{C}_{28} \mathrm{H}_{30} \mathrm{O}_{8} \mathrm{~S}$ [M+Na $]^{+}$: 549.1559; found: 549.1555.

\section{1,6-Anhydro-4-O-benzyl-2-O-\{(S)-2-(phenylthiomethyl)benzyl $\}-\beta$-D-}

glucopyranose (38). To a solution of $37(610 \mathrm{mg}, 1.16 \mathrm{mmol})$ in THF $(15 \mathrm{~mL})$ was added benzenethiol, sodium salt (512 $\mathrm{mg}, 90 \%, 3.48 \mathrm{mmol})$. The reaction mixture was stirred at room temperature for $2 \mathrm{~h}$, then concentrated in vacuo. The residue was dissolved with dichloromethane $(10 \mathrm{~mL})$ and washed with brine $(10 \mathrm{~mL})$. The organic phase was dried $\left(\mathrm{MgSO}_{4}\right)$, filtered and the filtrate was concentrated in vacuo. The residue was purified by silica gel column chromatography $(25 \%$ ethyl acetate in hexane) to afford $38(517 \mathrm{mg}, 96 \%)$ as colorless syrup, $R_{f}=0.56$ (ethyl acetate/hexane, 1/1); $[\alpha]^{20}{ }_{\mathrm{D}}=-37.23^{\circ}\left(c=4.0, \mathrm{CHCl}_{3}\right) ;{ }^{1} \mathrm{H} \mathrm{NMR}\left(500 \mathrm{MHz}, \mathrm{CDCl}_{3}\right)$ 
$\delta$ 7.36-7.16 (m,15H, aromatic), $5.52(\mathrm{~s}, 1 \mathrm{H}, \mathrm{H}-1), 4.61(\mathrm{~d}, 1 \mathrm{H}, J=12.0 \mathrm{~Hz}, \mathrm{CHHPh})$, $4.56(\mathrm{dd}, 1 \mathrm{H}, J=5.0,8.5 \mathrm{~Hz}, \mathrm{H}-7), 4.53-4.50(\mathrm{~m}, 1 \mathrm{H}, \mathrm{H}-4), 4.52(\mathrm{~d}, 1 \mathrm{H}, J=12.0 \mathrm{~Hz}$, CHHPh), 3.69-3.65 (m, 2H, H-8a, H-5), 3.61-3.59 (m, 1H, H-8b), 3.58 (dd, 1H, $J=$ 9.0, 14.0 Hz, H-3), 3.19-3.15 (m, 3H, H-2, H-6a, H-6b) $;{ }^{13} \mathrm{C}$ NMR (75 MHz, $\mathrm{CDCl}_{3}$ ) $\delta 140.64,137.89,136.31,129.40,129.01,128.72,128.46,128.44,127.82,127.78$, $126.83,101.42,81.33,80.79,80.24,75.64,71.71,71.12,66.83,41.74$; HR MALDITOF MS: m/z: calcd for $\mathrm{C}_{27} \mathrm{H}_{28} \mathrm{O}_{5} \mathrm{~S}$ [M+Na] $]^{+}$: 487.1555; found: 487.1540 .

\section{Acetyl 3,6-Di-O-acetyl-4-O-benzyl-2-O-\{(S)-2-(phenylthiomethyl)benzyl $\}-\alpha$-D-}

glucopyranose (39). Trimethylsilyl trifluoromethansulfonate $(2.2 \mu \mathrm{L}, 0.012 \mathrm{mmol})$ was added to a solution of $38(279 \mathrm{mg}, 0.60 \mathrm{mmol})$ in acetic anhydride $(10 \mathrm{~mL})$ at -50 ${ }^{\circ} \mathrm{C}$. The reaction mixture was allowed to warm to $-20{ }^{\circ} \mathrm{C}$ and then quenched with saturated aqueous $\mathrm{NaHCO}_{3}$, then extracted with $\mathrm{DCM}(2 \times 10 \mathrm{ml})$. The organic phase was washed with water $(10 \mathrm{ml})$ and brine $(10 \mathrm{ml})$ and dried $\left(\mathrm{MgSO}_{4}\right)$, filtered and the filtrate was concentrated in vacuo. The residue was purified by flash column chromatography (hexane/ethyl acetate $=3 / 1)$ to afford $39(329 \mathrm{mg}, 90 \%)$ as colorless syrup, $R_{f}=0.51$ (ethyl acetate/hexane, $\left.1 / 2\right) ;[\alpha]^{20}{ }_{\mathrm{D}}=-54.3^{\circ}\left(c=2.0, \mathrm{CHCl}_{3}\right) ;{ }^{1} \mathrm{H}$ NMR (300 MHz, $\left.\mathrm{CDCl}_{3}\right) \delta 7.379-7.16(\mathrm{~m}, 15 \mathrm{H}$, aromatic), $6.41(\mathrm{~d}, 1 \mathrm{H}, J=3.3 \mathrm{~Hz}, \mathrm{H}-$ 1), $5.51(\mathrm{t}, 1 \mathrm{H}, J=9.6 \mathrm{~Hz}, \mathrm{H}-3), 4.50(\mathrm{~d}, 1 \mathrm{H}, J=10.8 \mathrm{~Hz}, \mathrm{CHHPh}), 4.43(\mathrm{~d}, 1 \mathrm{H}, J=$ $10.8 \mathrm{~Hz}, \mathrm{CHHPh}$ ), 4.46 (m, 1H, H-7), 4.23-4.22 (m, 2H, H-6b, H-6a), 3.97-3.94 (m, 1H, H-5), 3.49-3.42 (m, 2H, H-2, H-4), 3.24 (dd, 1H, $J=8.4,14.0 \mathrm{~Hz}, \mathrm{H}-8 \mathrm{a}$ ), 3.03 (dd, $1 \mathrm{H}, J=5.1,14.0 \mathrm{~Hz}, \mathrm{H}-8 \mathrm{~b}), 2.14$ (s, 3H, $\mathrm{COCH}_{3}$ ), 2.02 (s, 3H, $\mathrm{COCH}_{3}$ ), 1.86 (s, $\left.3 \mathrm{H}, \mathrm{COCH}_{3}\right) ;{ }^{13} \mathrm{C} \mathrm{NMR}\left(75 \mathrm{MHz}, \mathrm{CDCl}_{3}\right) \delta 170.52,169.72,169.53,139.96,137.10$, 
$136.41,129.21,128.98,128.57,128.14,128.00,127.93,127.49,127.09,126.06$, $88.67,81.16,75.63,74.94,74.76,72.80,70.55,62.39,41.32,21.11,21.08,20.80 ; \mathrm{HR}$ MALDI-TOF MS: m/z: calcd for $\mathrm{C}_{33} \mathrm{H}_{36} \mathrm{O}_{9} \mathrm{~S}[\mathrm{M}+\mathrm{Na}]^{+}$: 631.2079; found: 631.2079 .

\section{3,6-Di-O-acetyl-4-O-benzyl-2-O-\{(S)-2-(phenylthiomethyl)benzyl\}- $\alpha$-D-}

glucopyranosyl trichloroacetimidate (8). Hydrazine acetate $(92 \mathrm{mg}, 1.0 \mathrm{mmol})$ was added to a solution of 39 (304 $\mathrm{mg}, 0.5 \mathrm{mmol})$ in $\mathrm{DMF}(5 \mathrm{~mL})$ at room temperature. The reaction mixture was stirred overnight and then quenched with saturated aqueous $\mathrm{NaHCO}_{3}$. The reaction mixture was extracted with ethyl acetate $(20 \mathrm{~mL})$. The organic phase was washed with saturated aqueous $\mathrm{NH}_{4} \mathrm{Cl}(20 \mathrm{~mL})$ and dried $\left(\mathrm{MgSO}_{4}\right)$, filtered and the filtrate was concentrated in vacuo. The residue was purified by silica gel column chromatography ( $25 \%$ ethyl acetate in hexane) to afford 3,6-di-O-acetyl-4-O-benzyl-2-O$\{(S)$-2-(phenylthiomethyl)benzyl $\}$-D-glucopyranose $\quad(7, \quad 263 \quad \mathrm{mg}, \quad 93 \%)$. Trichloroacetonitrile $(0.46 \mathrm{~mL}, 4.6 \mathrm{mmol})$ and 1,8-diazabicyclo[5.4.0]undec-7-ene (DBU) $(28 \mu \mathrm{L}, 0.19 \mathrm{mmol})$ were added to a solution of $7(263 \mathrm{mg}, 0.46 \mathrm{mmol})$ in dichloromethane $(5 \mathrm{~mL})$ at $0{ }^{\circ} \mathrm{C}$. The reaction mixture was stirred at the same temperature for $1 \mathrm{~h}$ and then concentrated in vacuo. The residue was purified by silica gel column chromatography (20\% ethyl acetate in hexane) to afford 8 (300 mg, 91\%): $R_{f}=0.45$ (dichloromethane/acetone, 100/1); $[\alpha]^{20}{ }_{\mathrm{D}}=-0.03^{\circ}\left(c=6.0, \mathrm{CHCl}_{3}\right) ;{ }^{1} \mathrm{H} \mathrm{NMR}(300 \mathrm{MHz}$, $\left.\mathrm{CDCl}_{3}\right) \delta 8.61(\mathrm{~s}, 1 \mathrm{H}, \mathrm{N} H), 7.37-7.16(\mathrm{~m}, 15 \mathrm{H}$, aromatic), $6.62(\mathrm{~d}, 1 \mathrm{H}, J=3.6 \mathrm{~Hz}, \mathrm{H}-1)$, 5.57 (t, $1 \mathrm{H}, J=9.6 \mathrm{~Hz}, \mathrm{H}-3), 4.52-4.42$ (m, 1H, H-7), 4.50 (d, $1 \mathrm{H}, J=10.5 \mathrm{~Hz}, \mathrm{CHHPh}$ ), 4.44 (d, 1H, $J=10.5 \mathrm{~Hz}, \mathrm{CHHPh}$ ), 4.29-4.17 (m, 2H, H-6a, H-6b), 4.13-4.08 (m, 1H, H5), 3.55 (dd, 1H, $J=3.6,9.6 \mathrm{~Hz}, \mathrm{H}-2), 3.49$ (t, $1 \mathrm{H}, J=9.6 \mathrm{~Hz}, \mathrm{H}-4), 3.25$ (dd, $1 \mathrm{H}, J=$ 
6.9, $13.5 \mathrm{~Hz}, \mathrm{H}-8 \mathrm{a}$ ), 3.05 (dd, $1 \mathrm{H}, J=6.6,13.5 \mathrm{~Hz}, \mathrm{H}-8 \mathrm{~b}$ ), 2.00 (s, 3H, $\mathrm{COCH}_{3}$ ), 1.81 (s, $\left.3 \mathrm{H}, \mathrm{COCH}_{3}\right) ;{ }^{13} \mathrm{C} \mathrm{NMR}\left(75 \mathrm{MHz}, \mathrm{CDCl}_{3}\right) \delta 170.44,169.49,161.08,139.80,137.07$, $136,21,129.35,128.97,128.54,128.53,128.13,127.22,126.11,93.07,80.86,75.51$, 75.47, 74.54, 72.70, 70.96, 62.33, 41.27, 20.95, 20.77; HR MALDI-TOF MS: m/z: calcd for $\mathrm{C}_{33} \mathrm{H}_{34} \mathrm{Cl}_{3} \mathrm{NO}_{8} \mathrm{~S}[\mathrm{M}+\mathrm{Na}]^{+}:$732.0968; found: 732.0957 . 


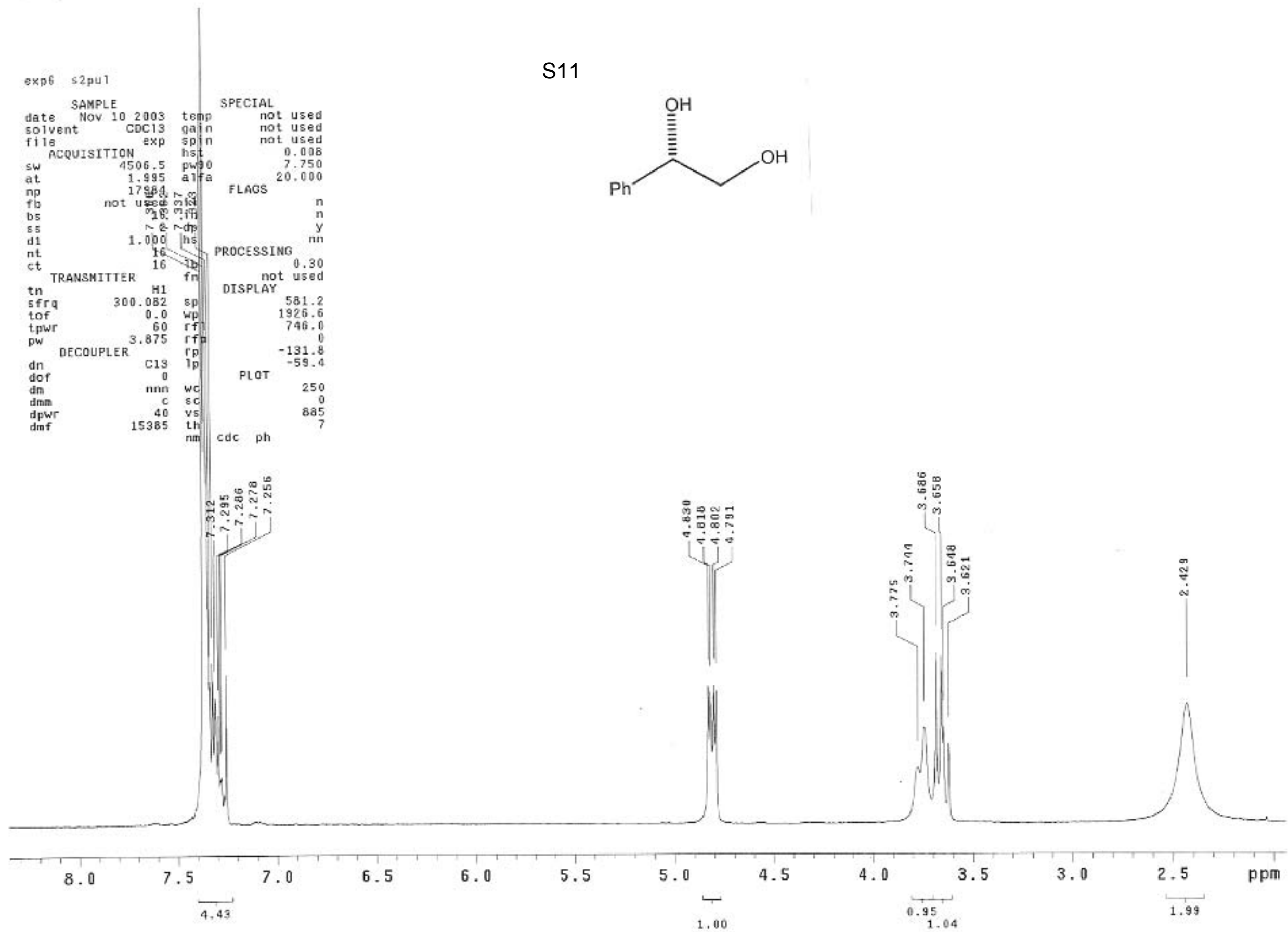




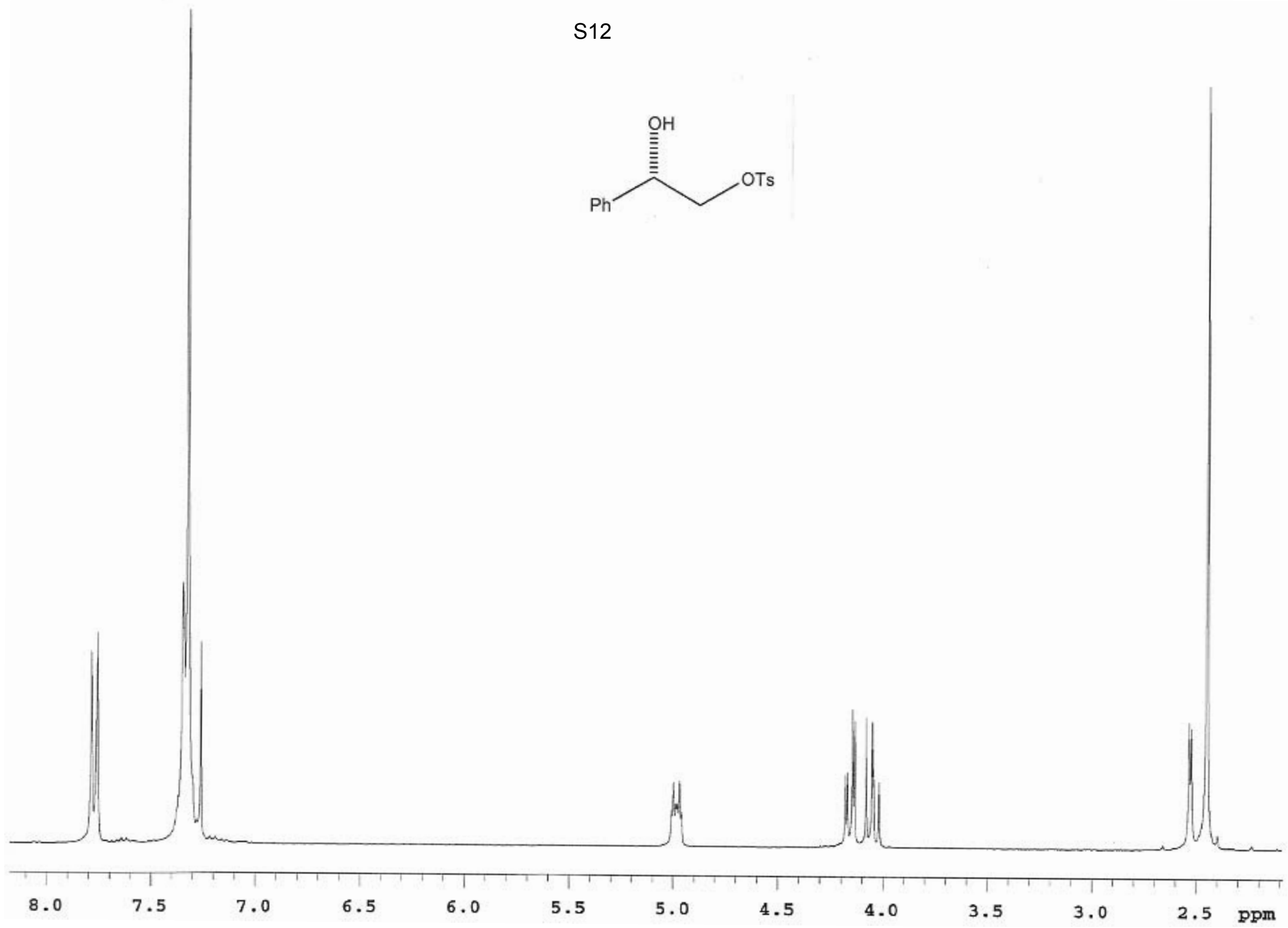




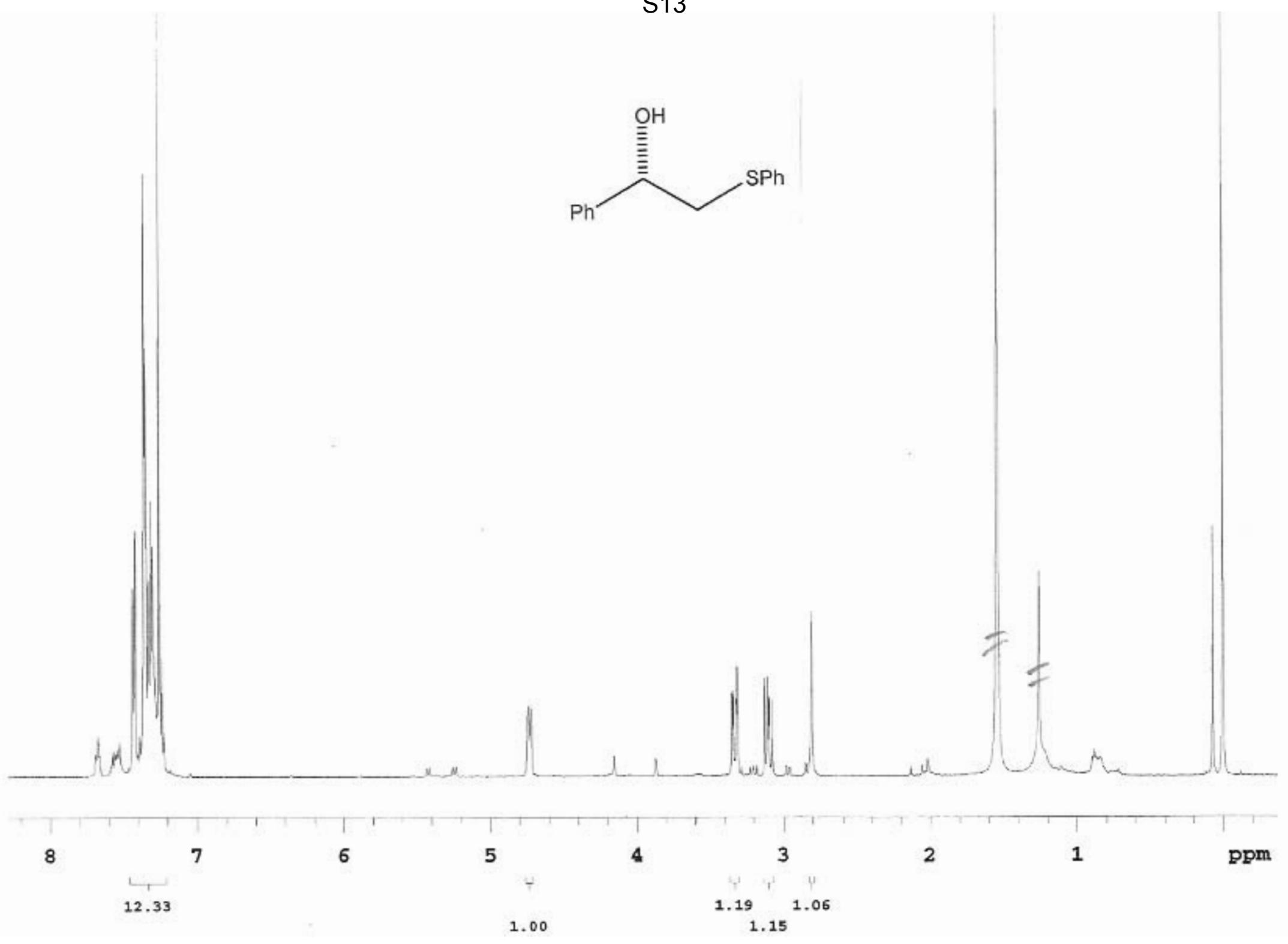




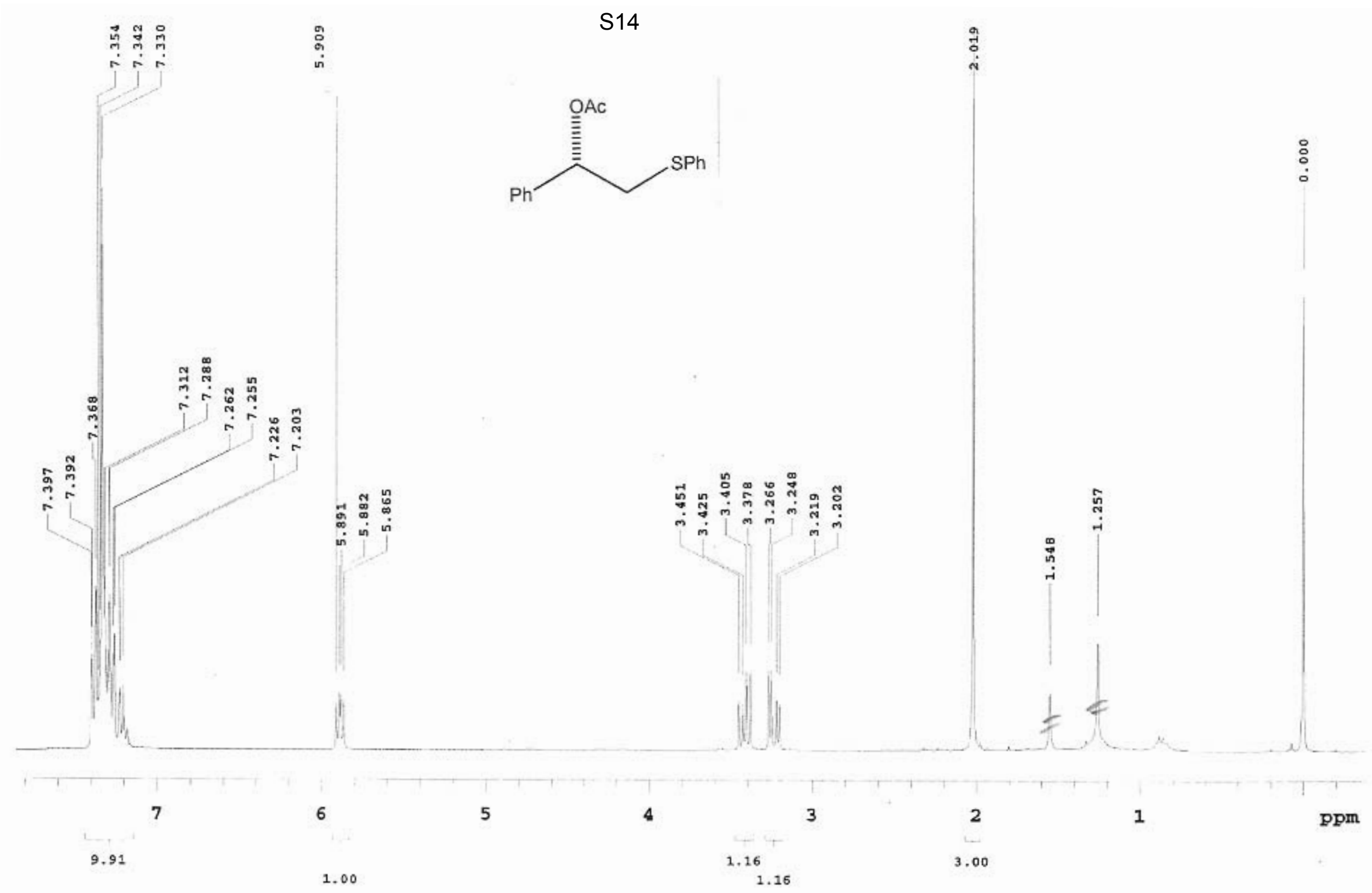


Archive directory: /export/home/hyang/vnmrsys/data

Sample directory: /export/home/hyang/vnarsys/data/hyang_29Nov2003

Pulse Sequence: $\$ 20 u 7$

Solvent: $\mathrm{CDC} 1 \mathrm{~s}$

Temp. $25.0 \mathrm{C} / 298.1 \mathrm{~K}$
Hercury- $300 \mathrm{BB}$
"merc $300^{\circ}$

Relax. de lay $1.000 \mathrm{sec}$

Pulse 45.0 degrees

Acq. ${ }^{2}$ time 1,995 sec

64 repetitions

OBSERVE H1, $300.0803986 \mathrm{MHZ}$

DATA PROCESSINC

Line broadening $0.3 \mathrm{~Hz}$

Total time $3=i n, 27$ sec
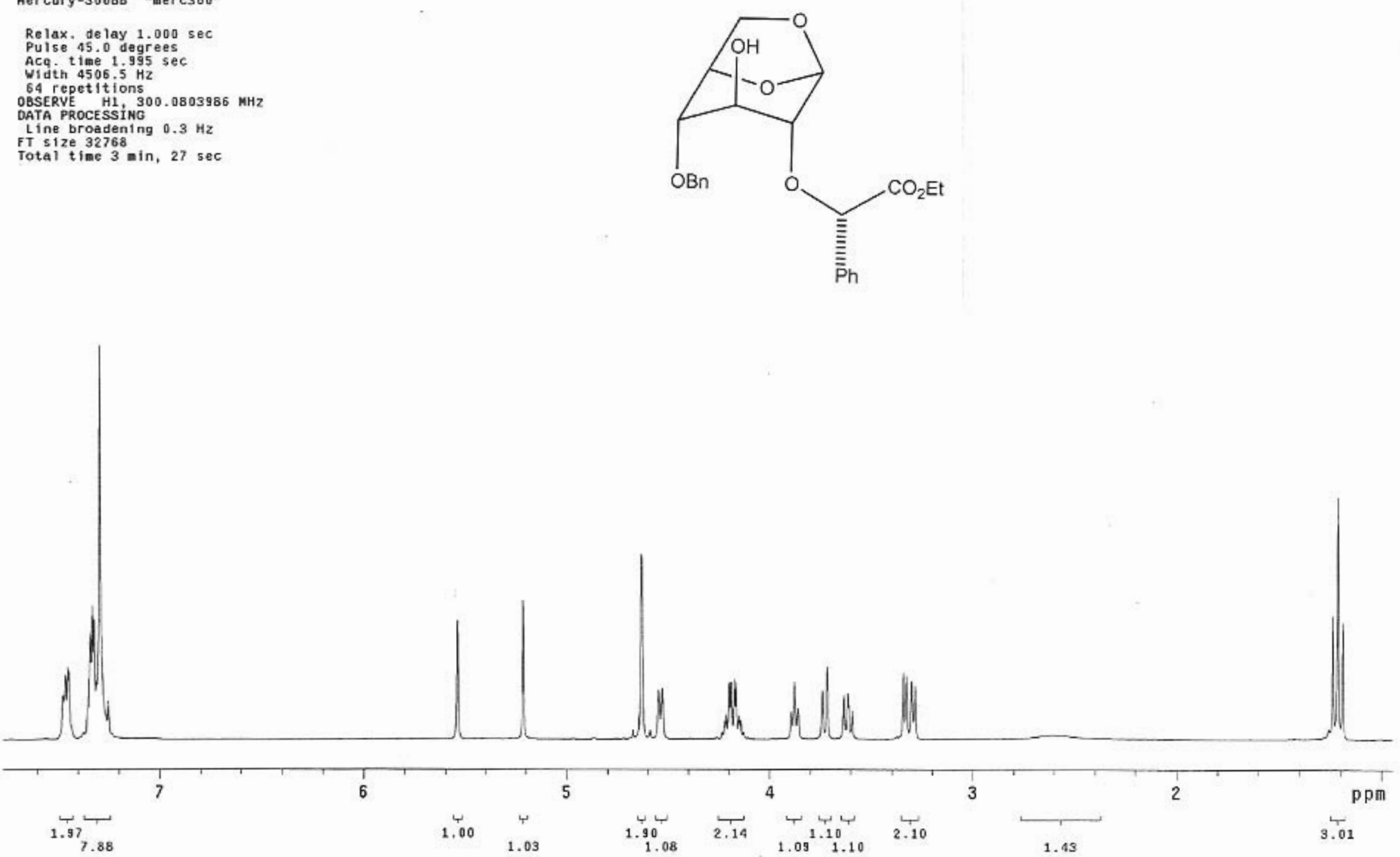


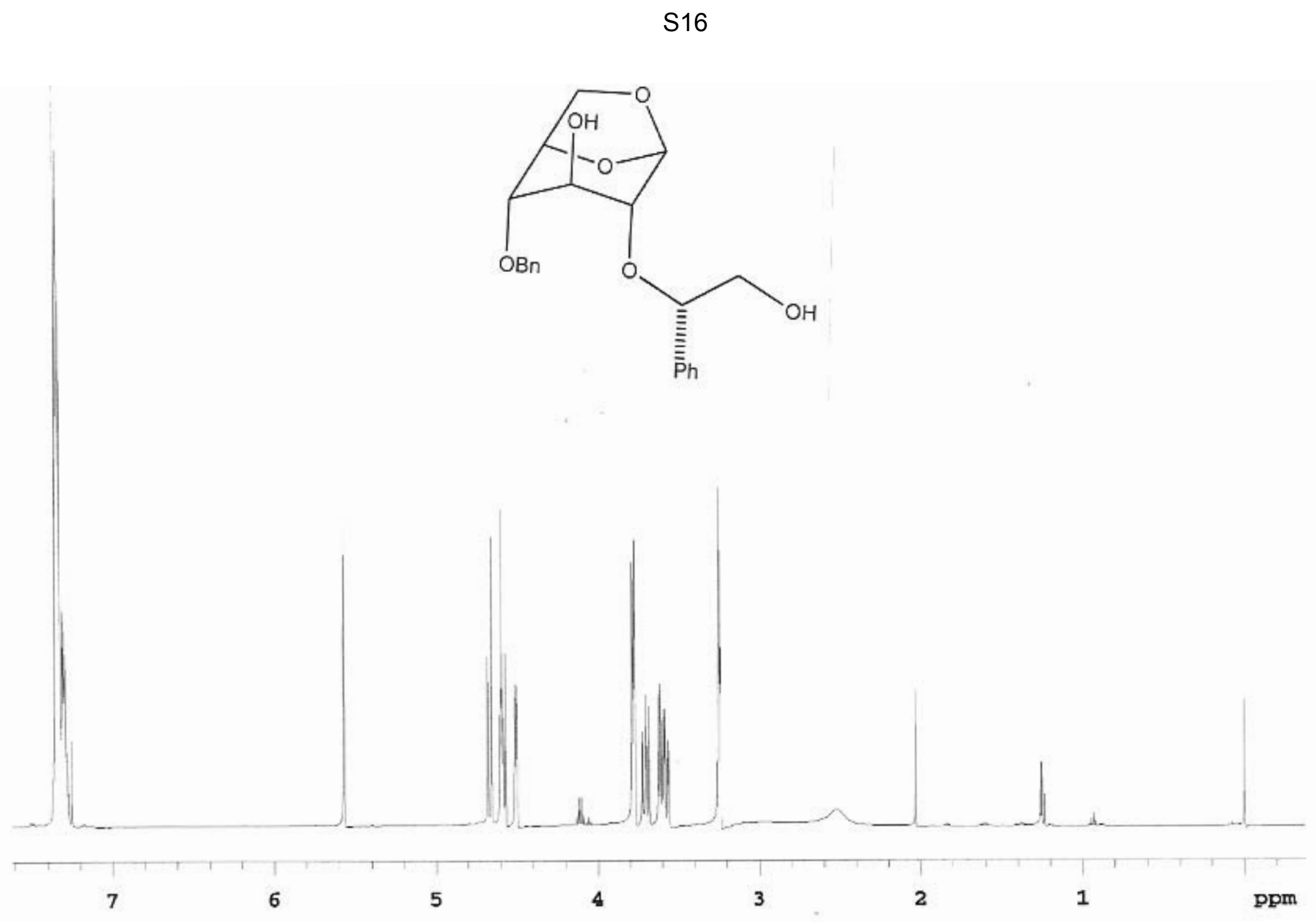




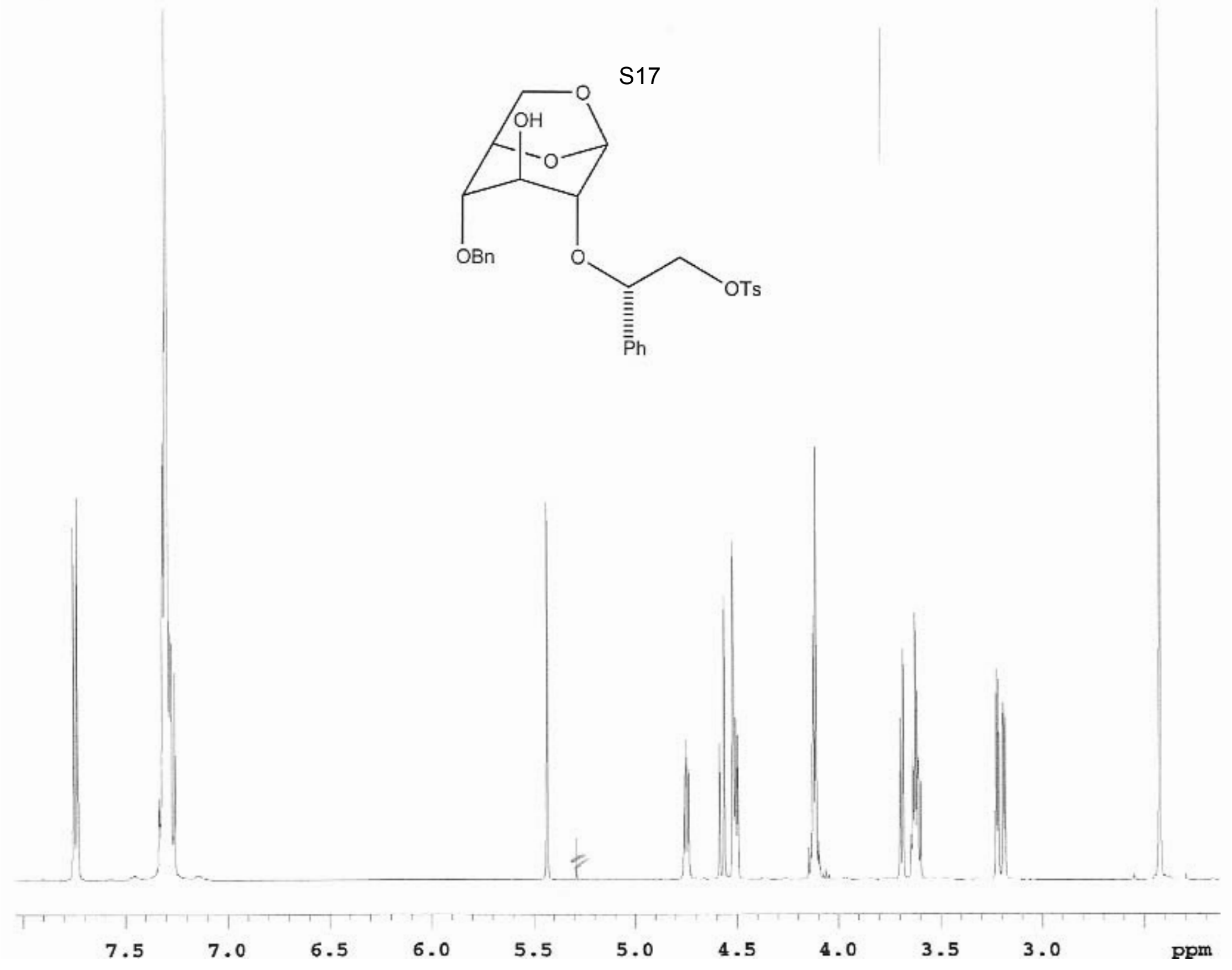



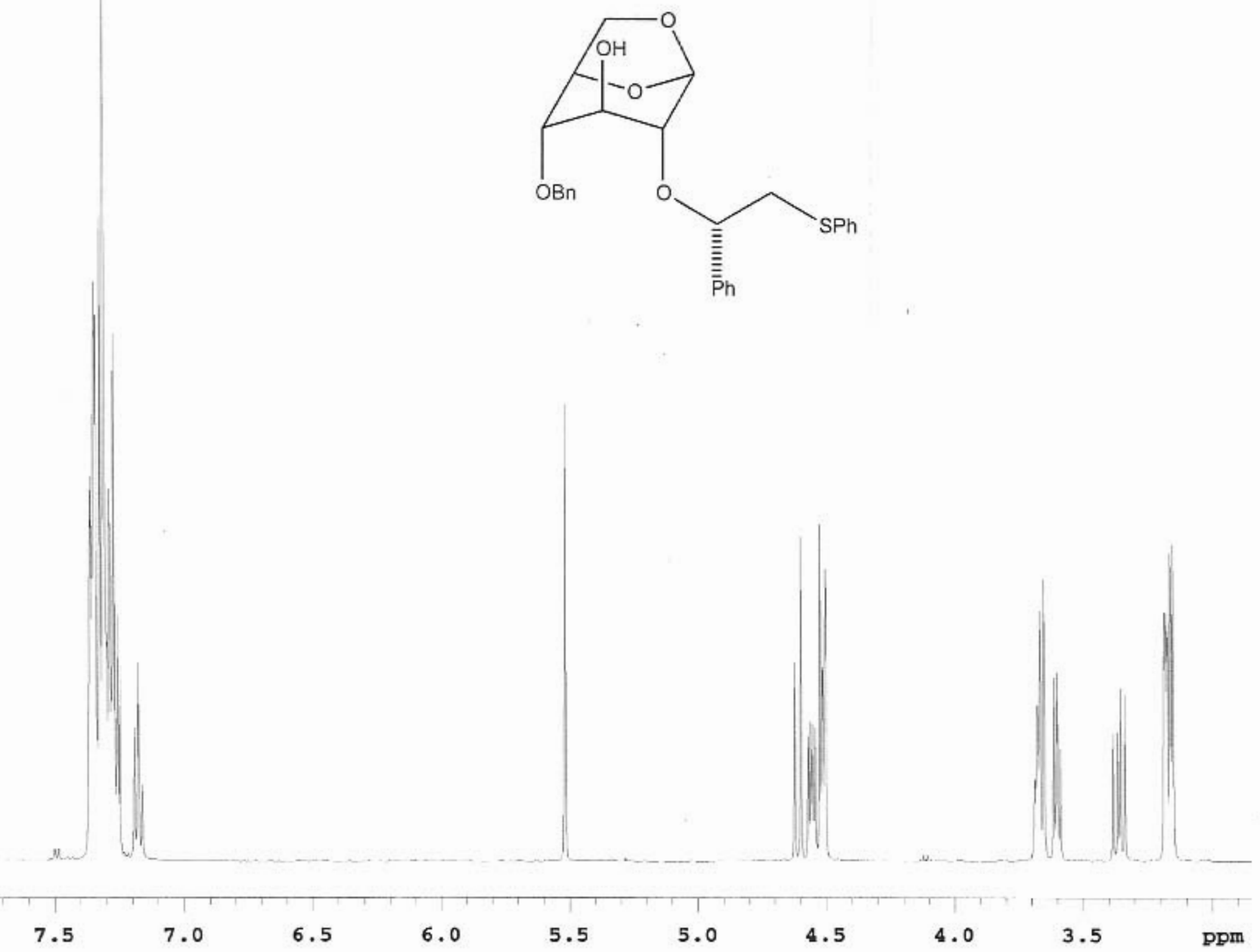


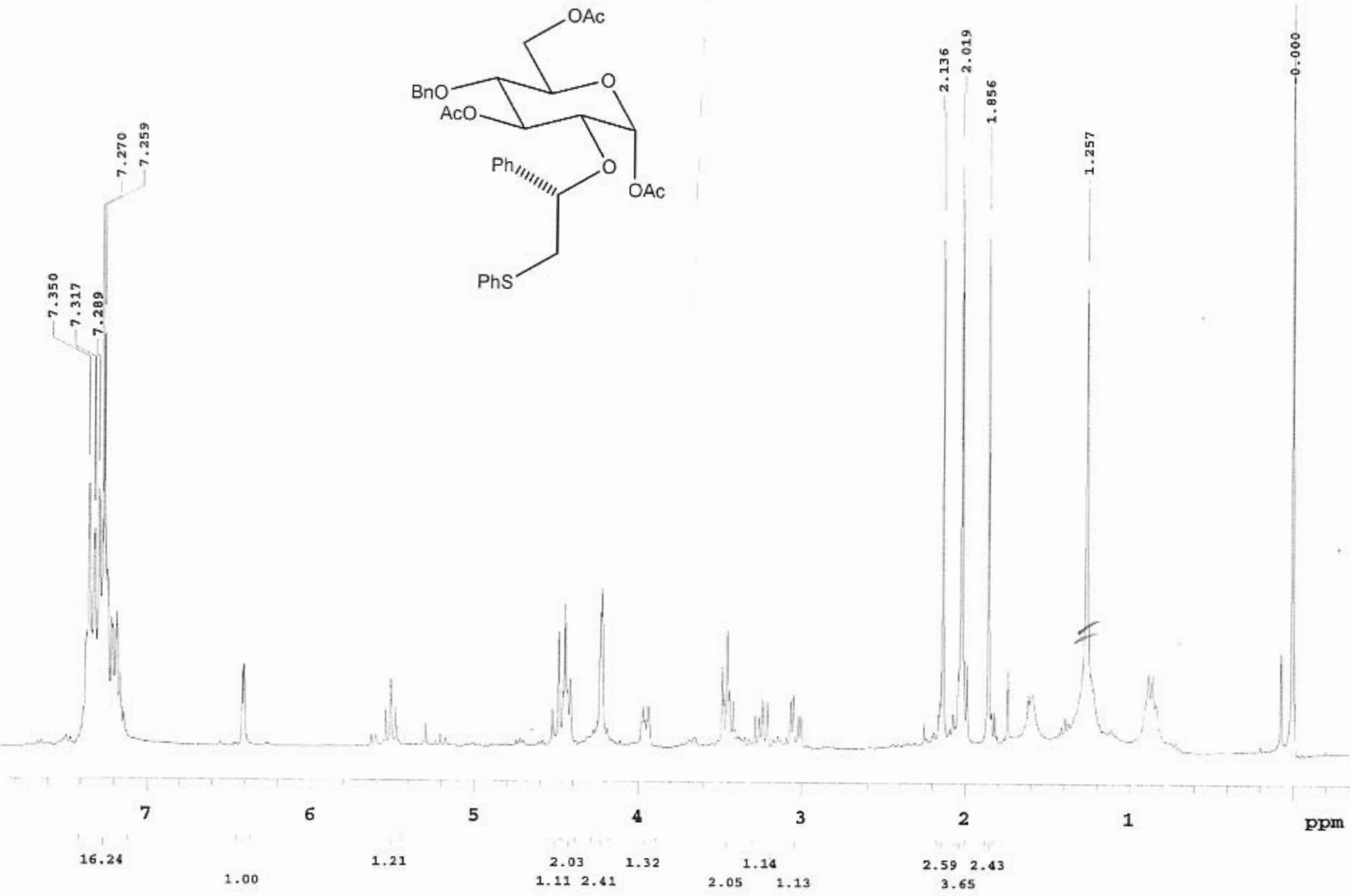


S20

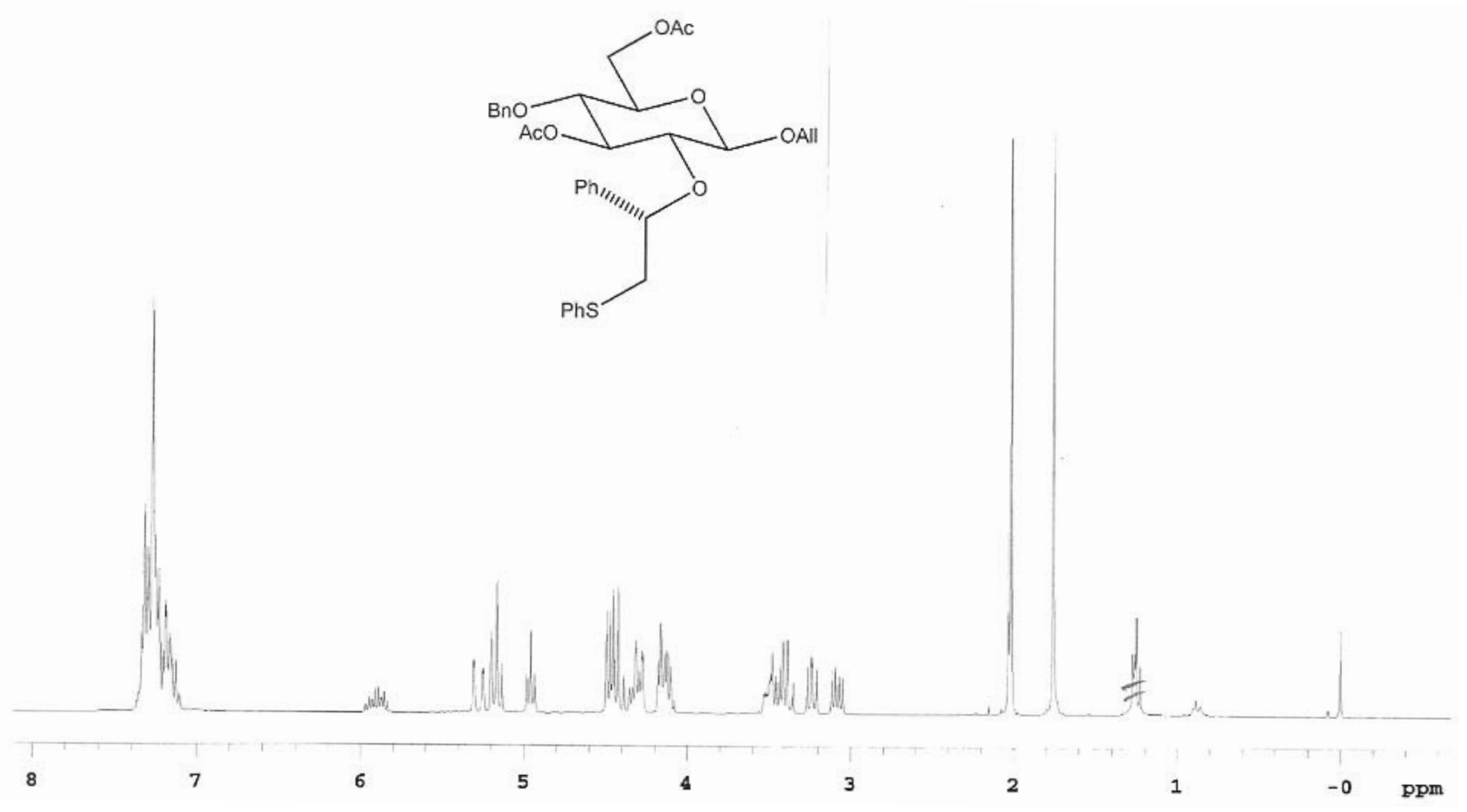




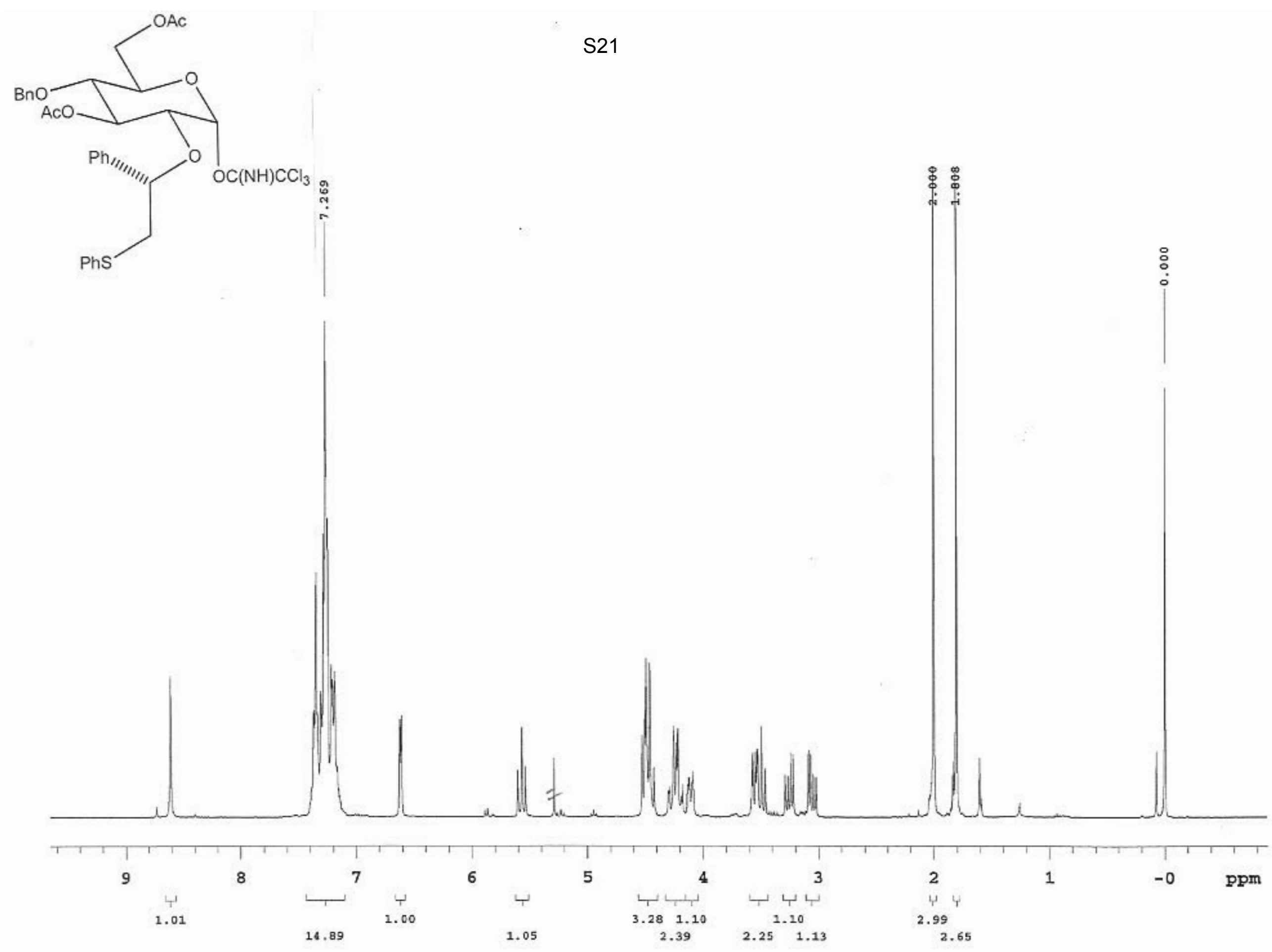




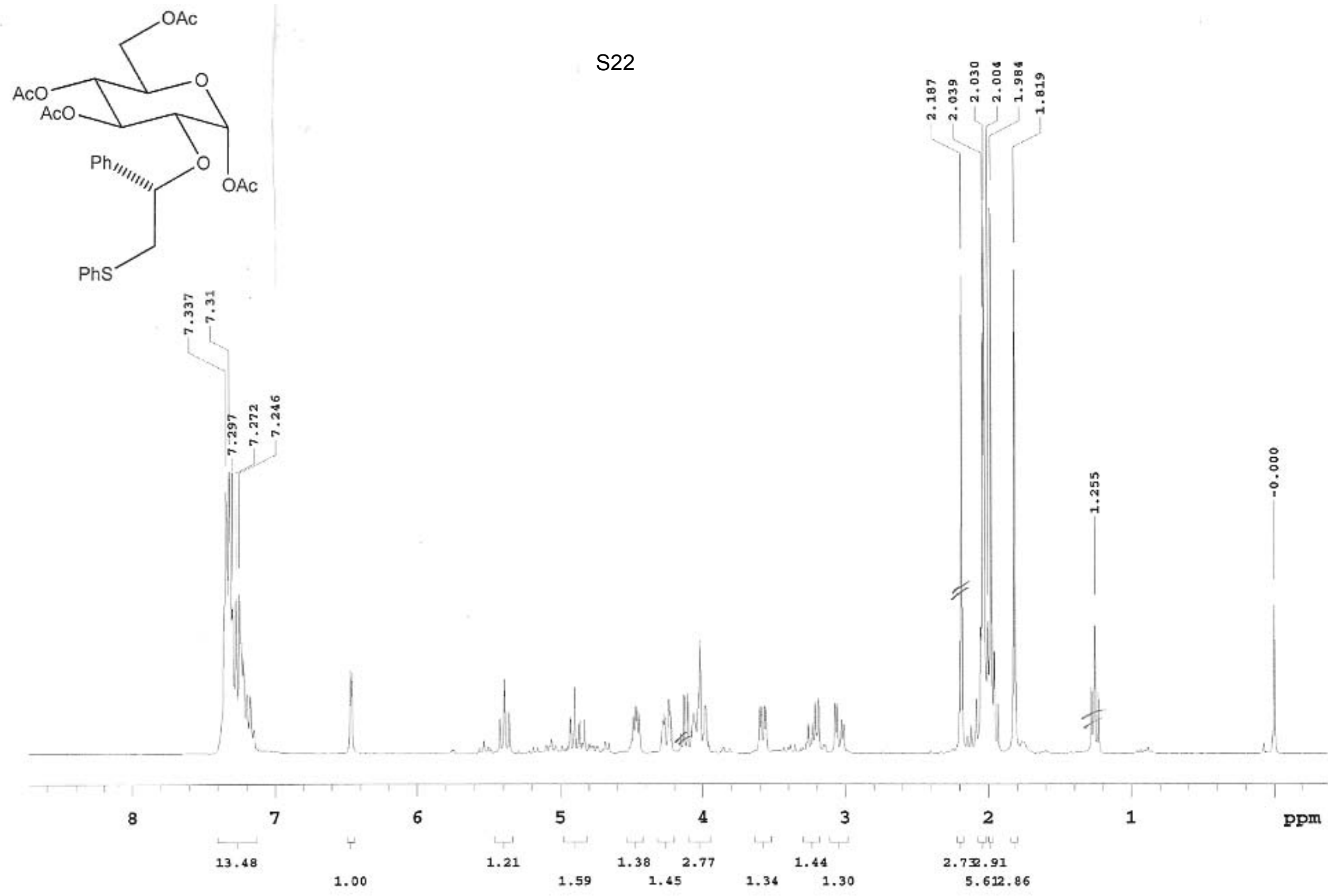




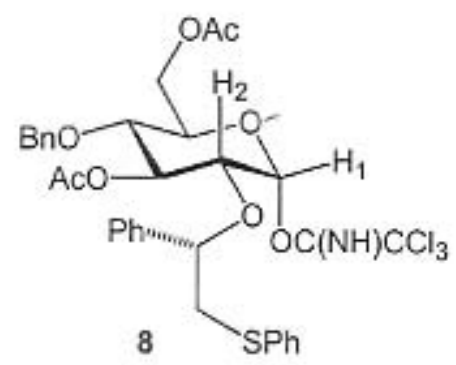

S24

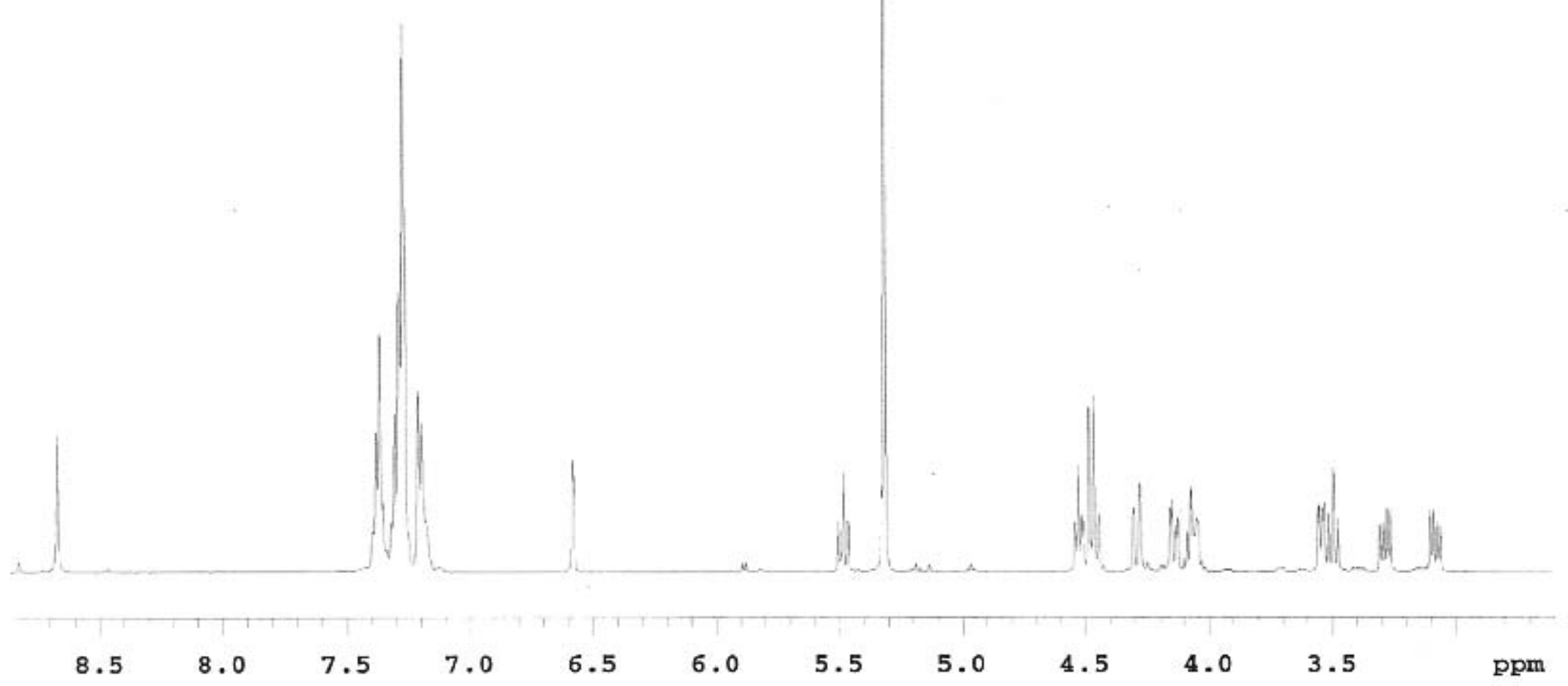




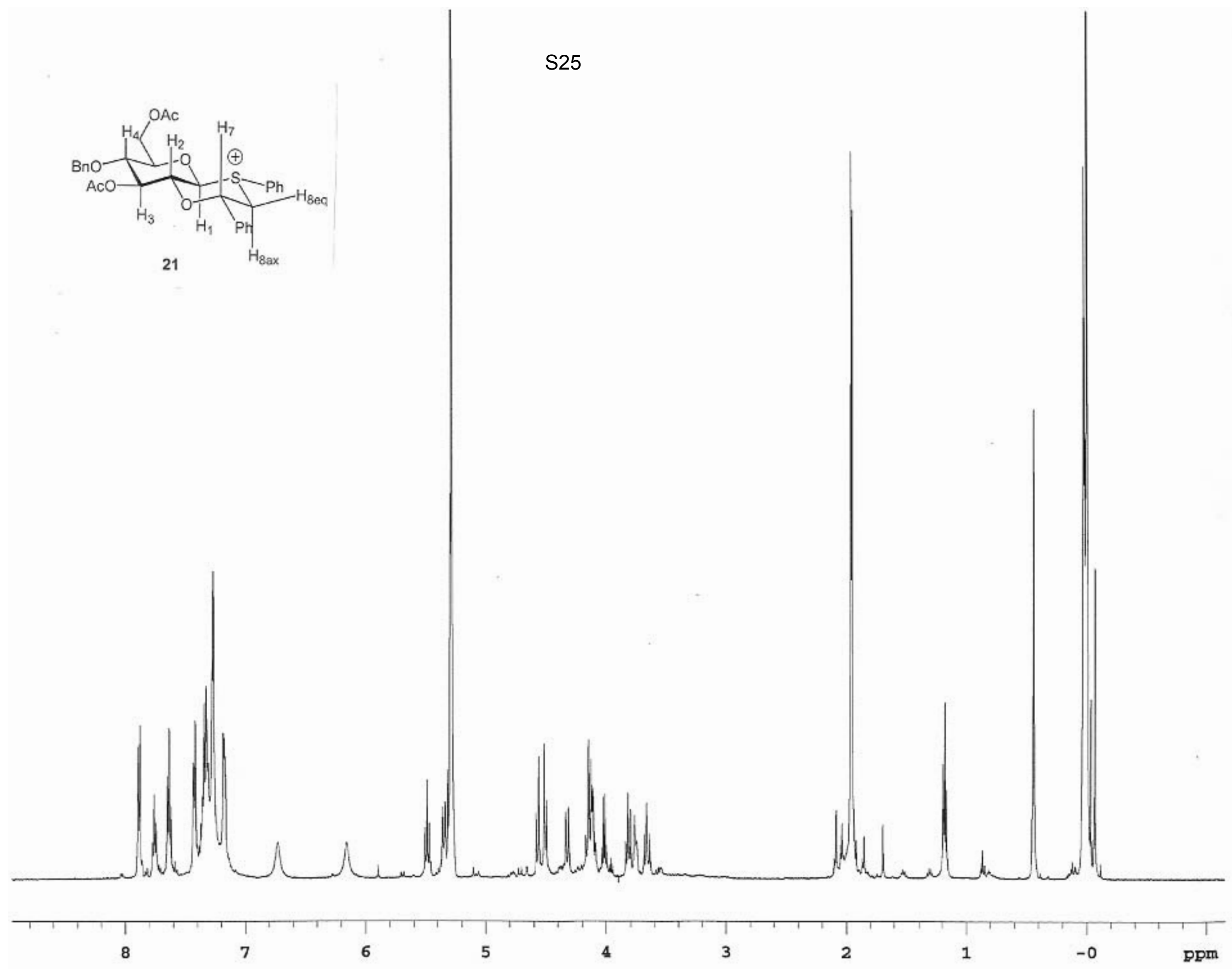


${ }^{1} \mathrm{H}$ TOCSY $1 \mathrm{D}$ spectrum on irradiation of $\mathrm{H} 4$ of sulfonium ion 21

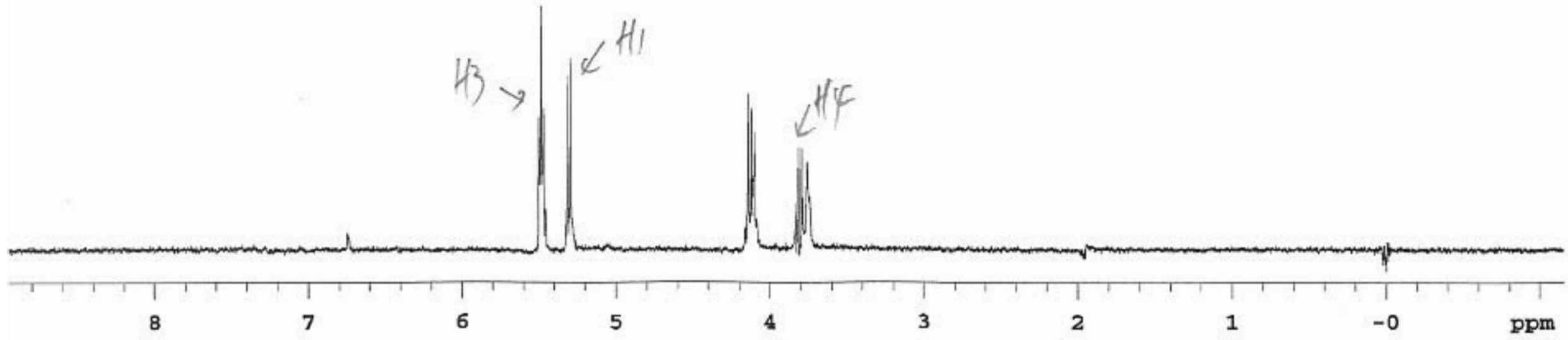


${ }^{1} \mathrm{H}$ TOCSY 1D spectrum on irradiation of $\mathrm{H} 7$ of sulfonium ion 21

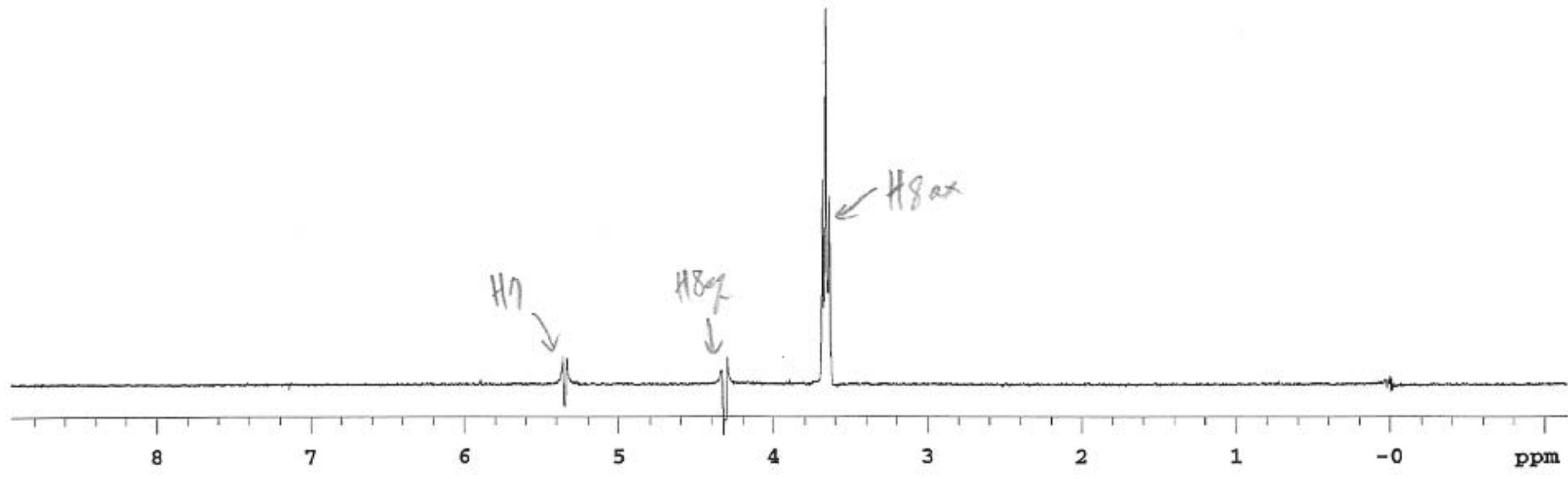


Archive directory: /export/home/glushlea/vanrays/data

Sample directory: /export/home/glushka/vnmrsys/data/glushka_gHSQC_13Aug2004

\section{Pulse Sequence: gHsQC}

Solvent: $\mathrm{CD} 2 \mathrm{Cl2}$

Temp. $-30.0 \mathrm{C} / 243.2 \mathrm{~K}$

File: kim?_hsge

IMOVR-600 "nmr1"

Relax. delay 1,000 sec Acq. time $0.200 \mathrm{gec}$

width $5049.2 \mathrm{~Hz}$

2D width $20115.7 \mathrm{~Hz}$

2 repetitions

$2 x$ 64 increments

OBSERVE H1, $499.9729949 \mathrm{MHz}$ DECOUPLE C13, $125.7283289 \mathrm{MHz}$ Power $47 \mathrm{~dB}$

on during acquisition

off during delay

GARP-1 modulated

DATA PROCESSING

Gauss apodization $0.092 \mathrm{sec}$

F1 DATA PROCESSING

Gauss apodization 0.003 sec

FT вize $2048 \times 512$

Total time $5 \mathrm{~min}, 17 \mathrm{sec}$

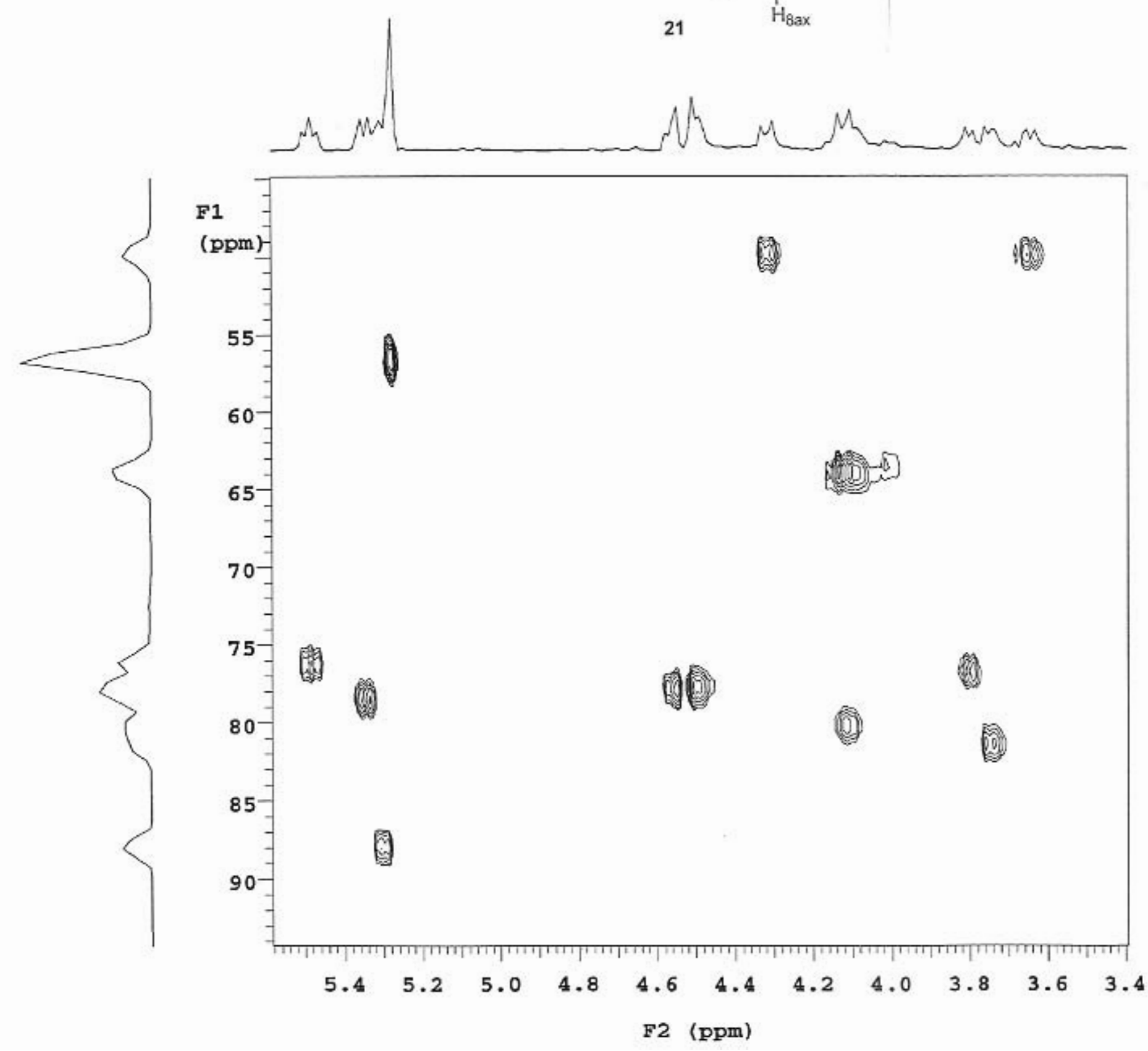


Archive airectory: /export/home/glushka/vnmrsys/data

Sample directory: /export/home/glushka/vnmrtys/data/gluehka_gHSQC_13nug2004

Pulse Sequence: crisisghrac

Solvent: $\mathrm{CD} 2 \mathrm{C} 12$

Temp. $-30.0 \mathrm{C} / 243.2 \mathrm{~K}$

File: kim10_hmbc

ImOVR-600 "nar1

Relax. delay 1.797 sec

Acq. time $0.203 \mathrm{sec}$

Wiath $5049.2 \mathrm{~Hz}$

2D width $7542.9 \mathrm{~Hz}$

4 repetitions

64 increments

OBSERVE H1, 499.9729949 MHz

DATA PROCESSING

Gauss apodization $0.094 \mathrm{sec}$

F1 DATX PROCESSING

Gauss apodization $0.008 \mathrm{sec}$

FT 8ize $2048 \times 512$

Total time 0 min, -1 sec

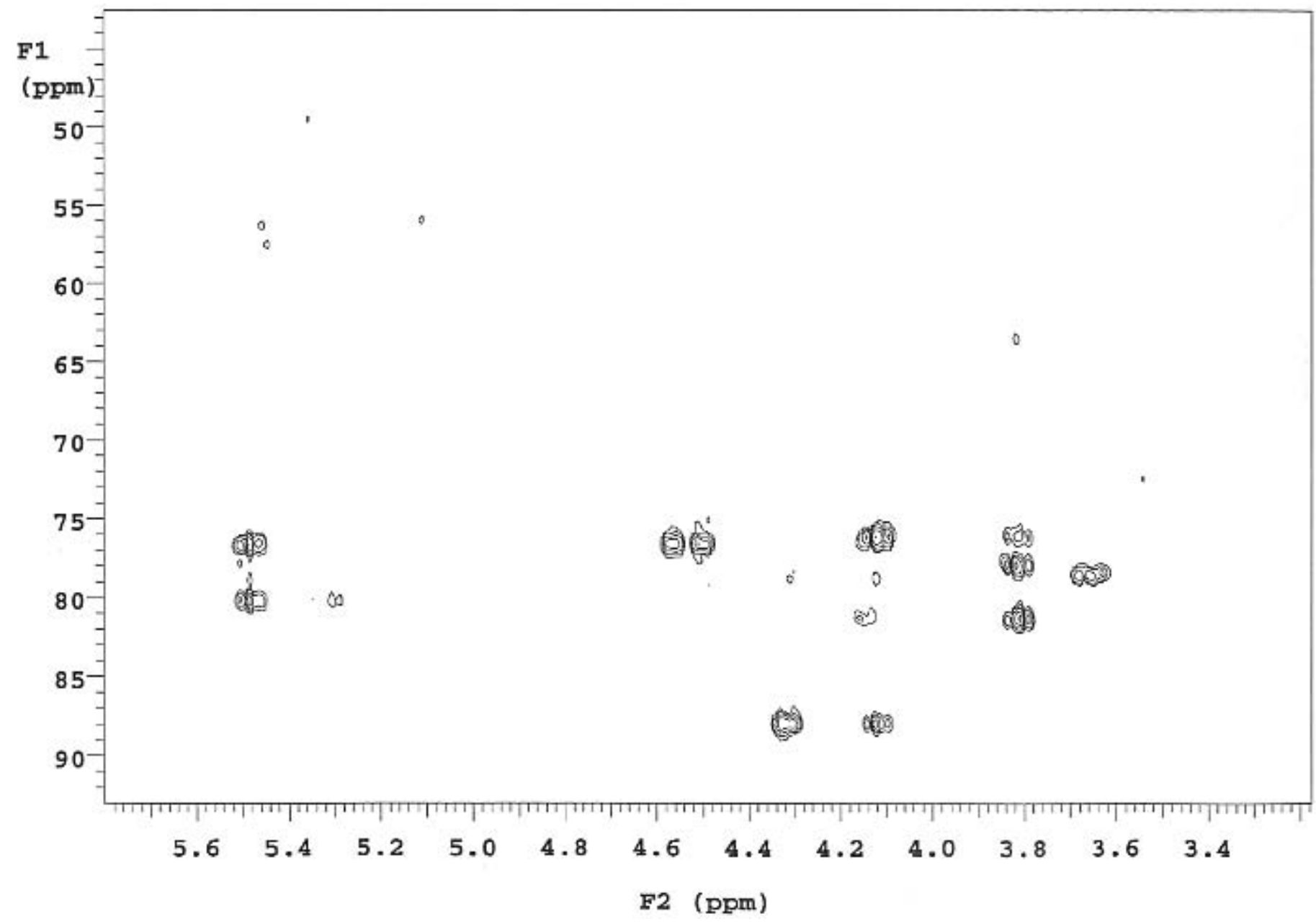



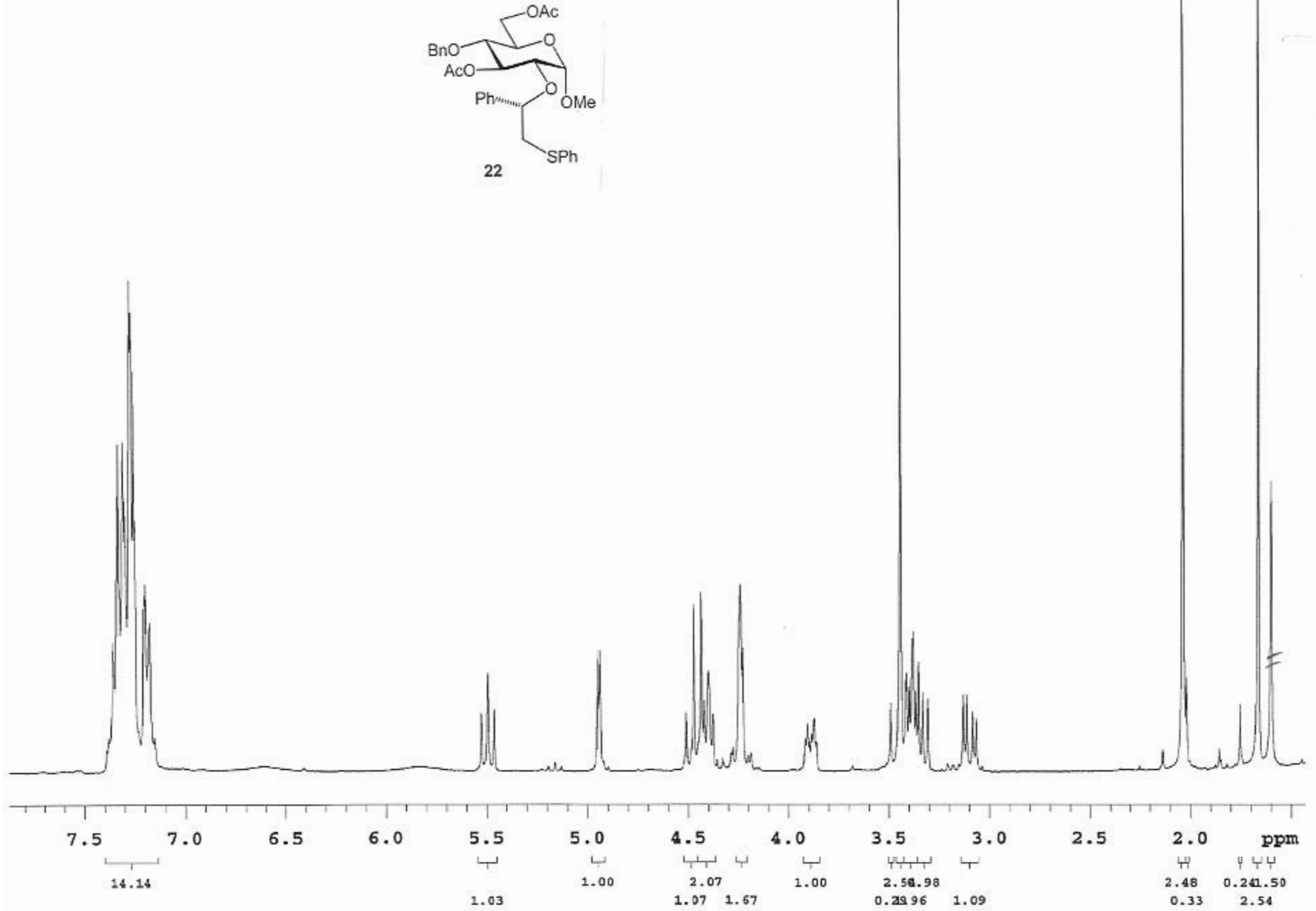


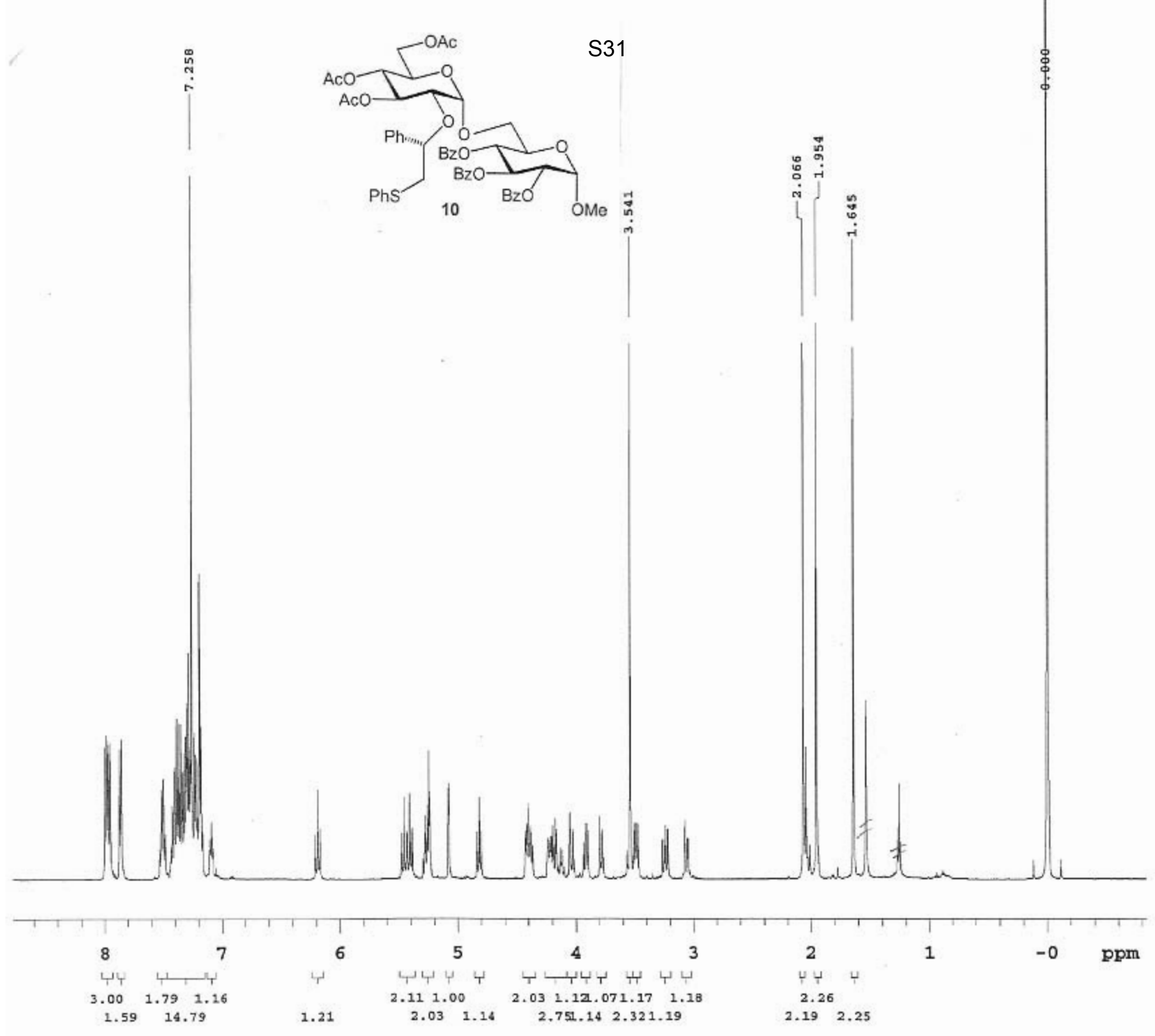




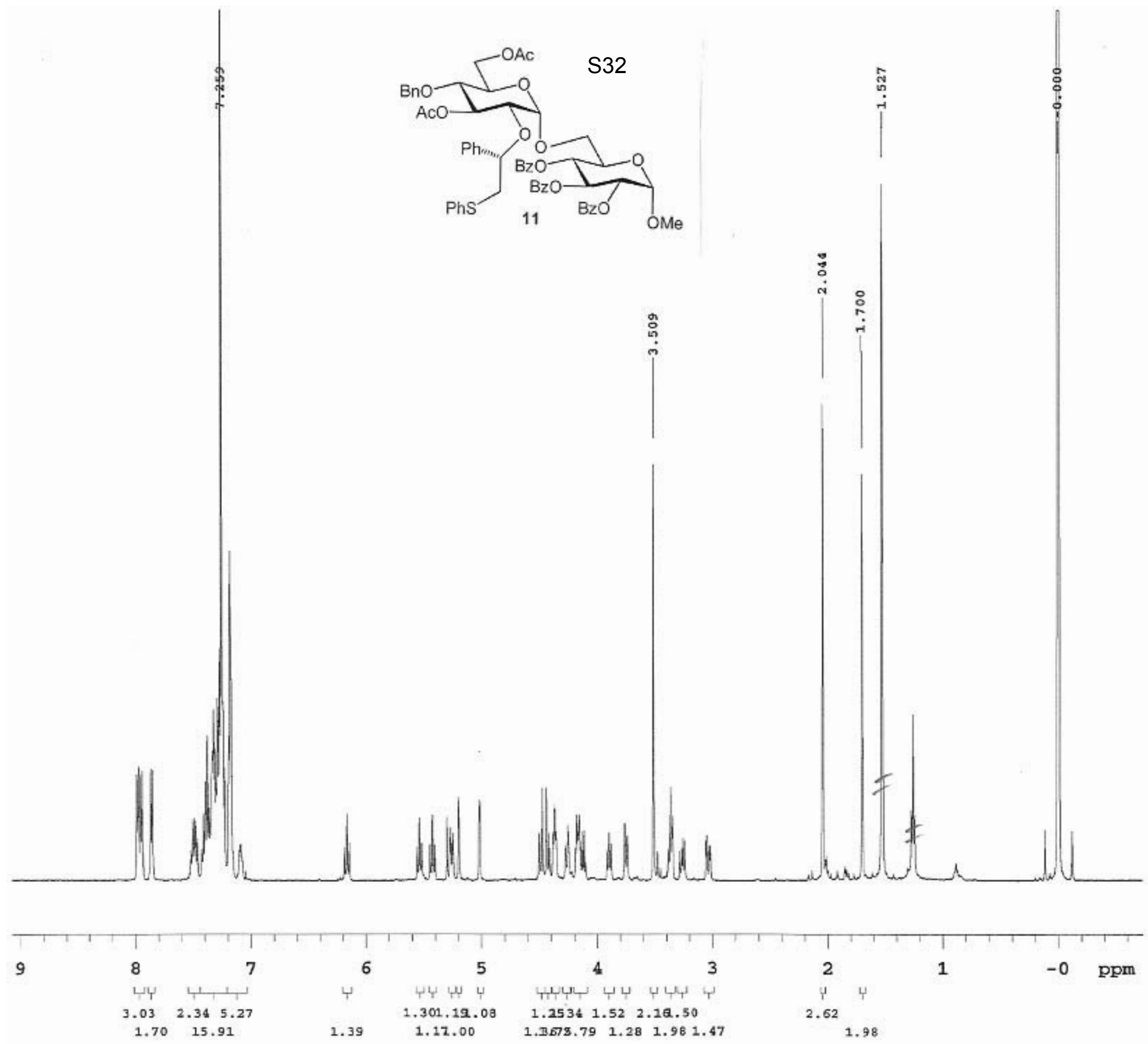


S33
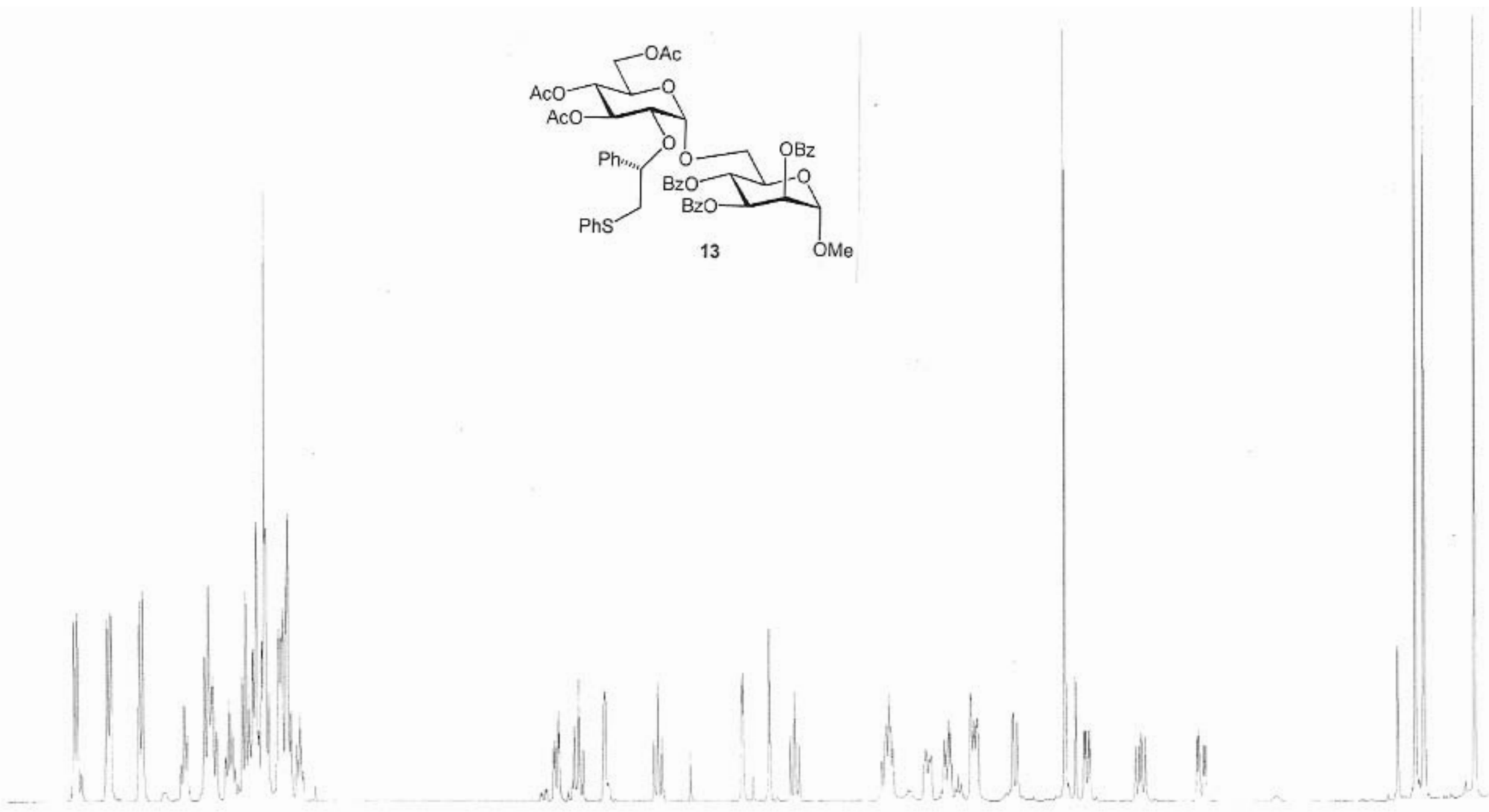

8

7

6

5

4

3

2 ppm 
S34
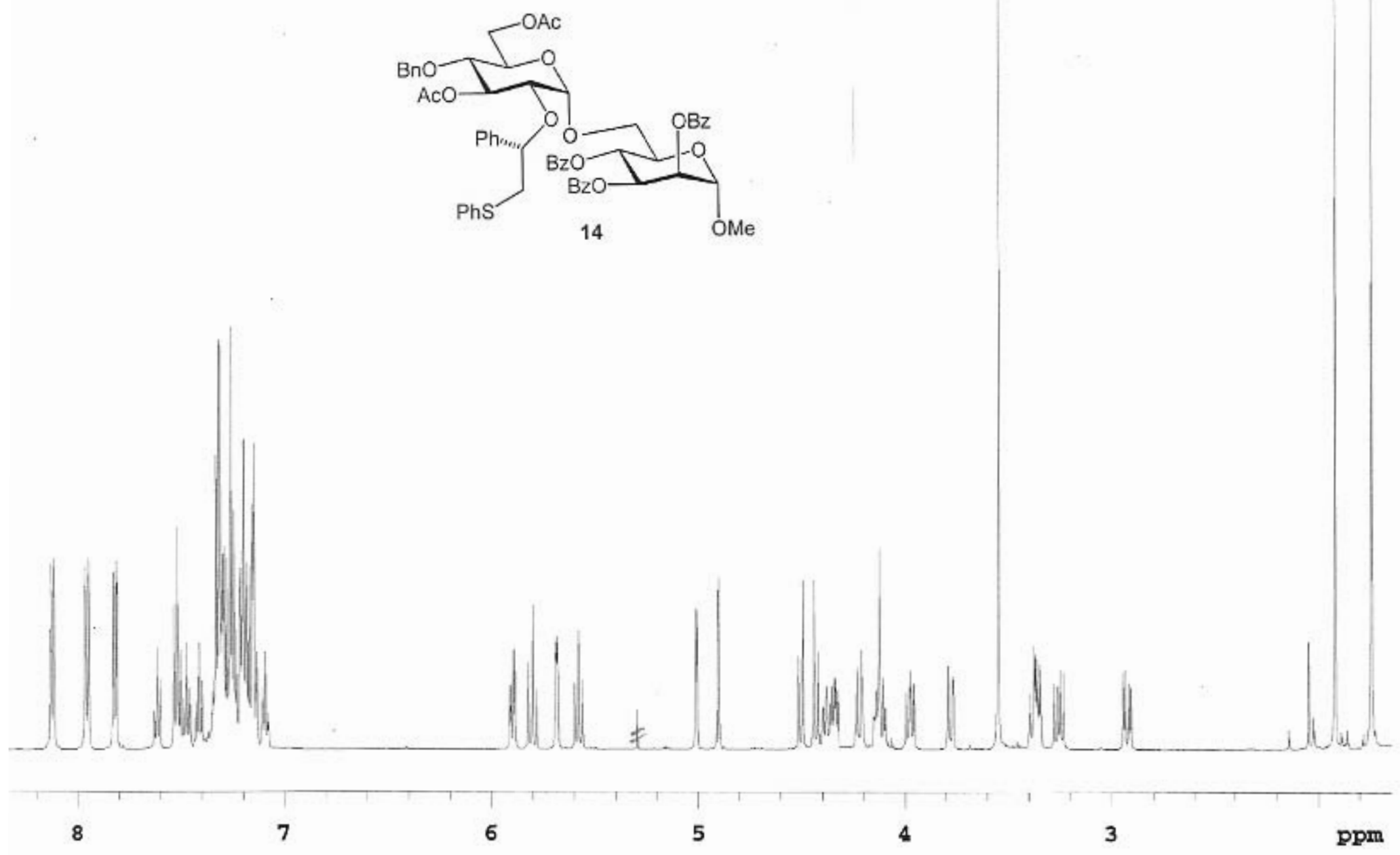

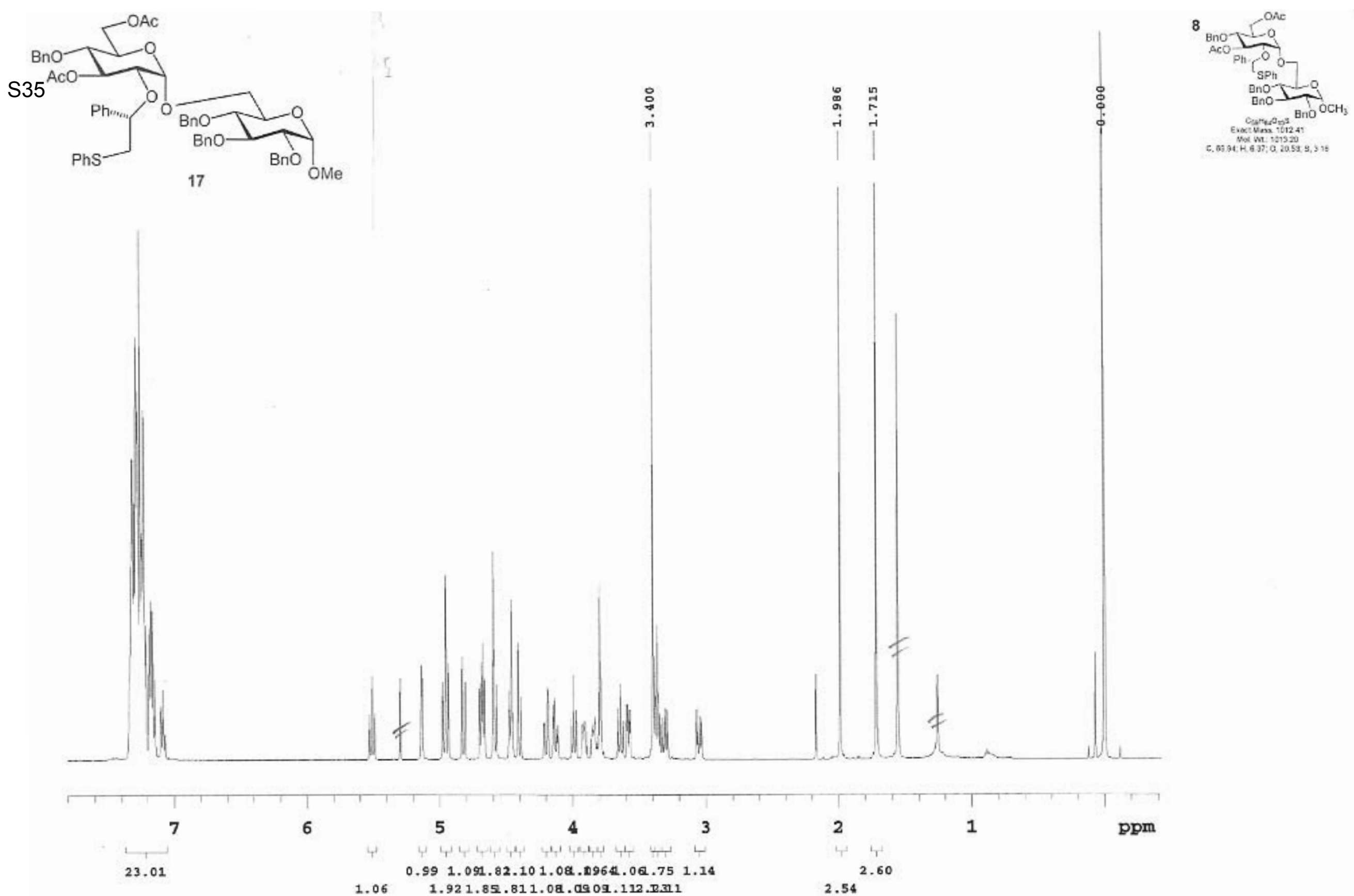


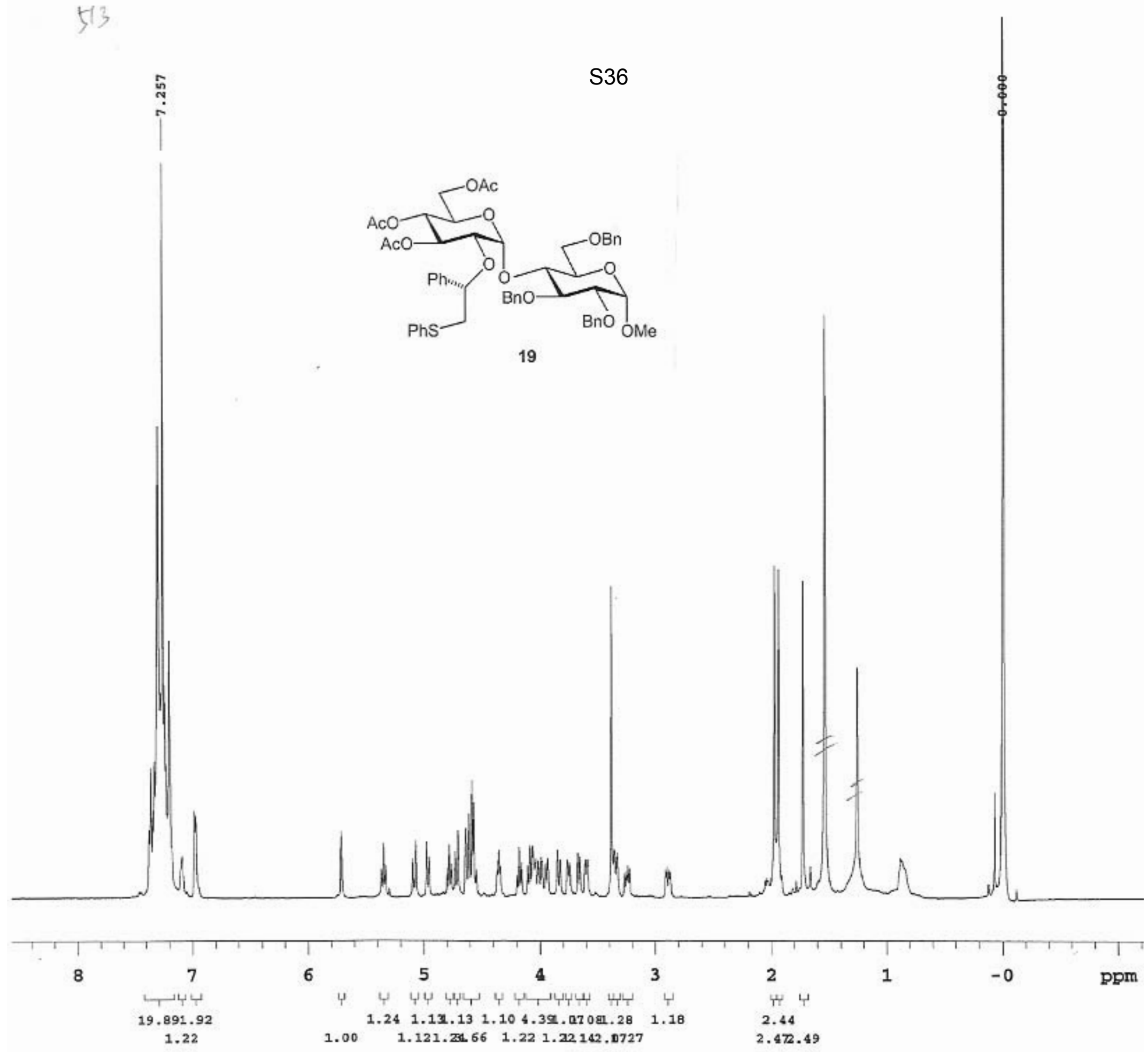




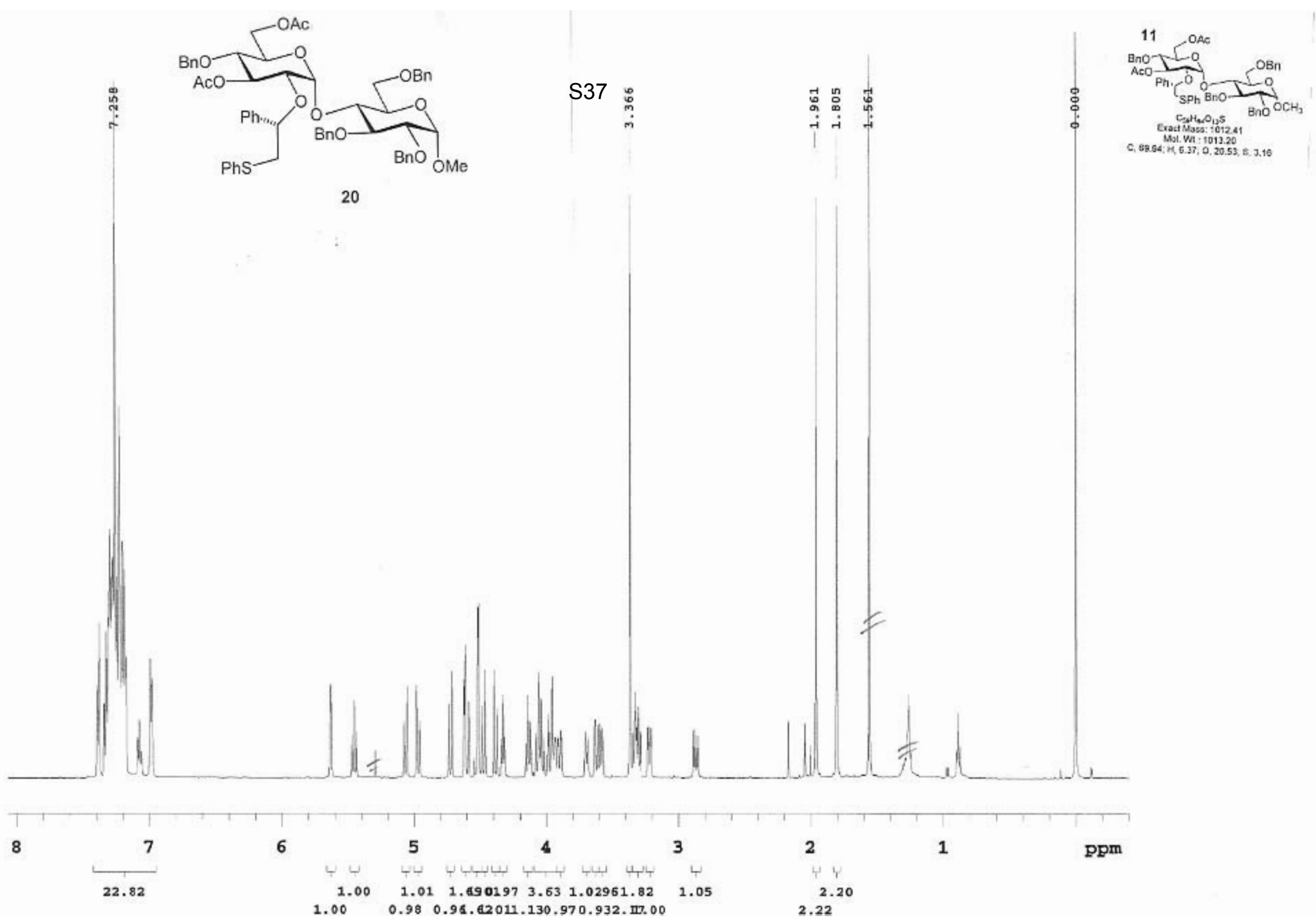




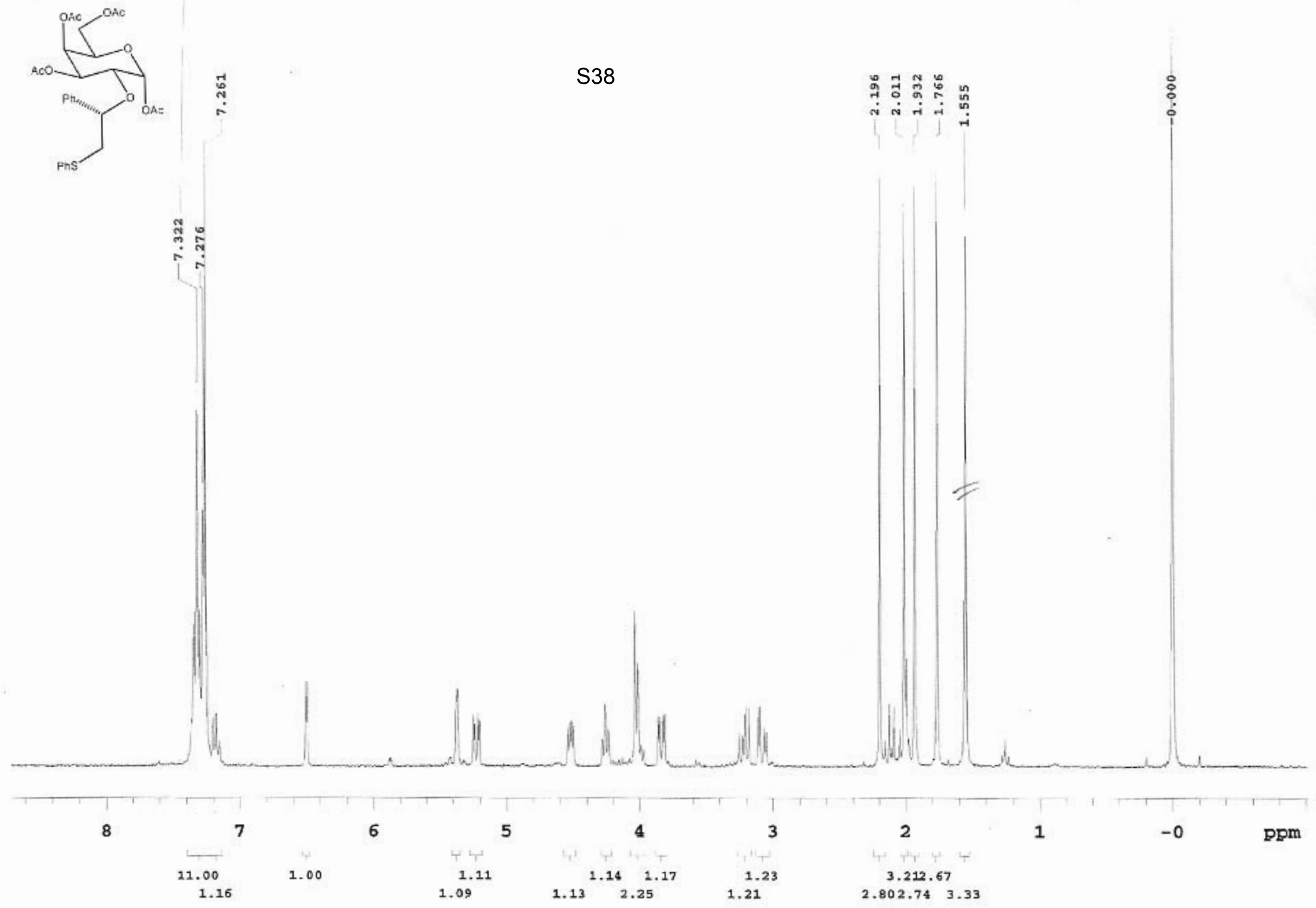


S39

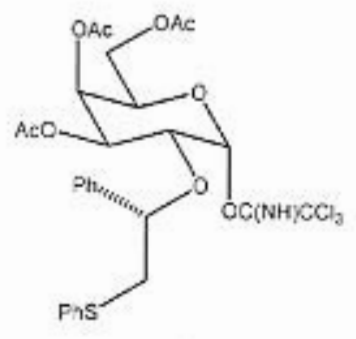

$13 \mathrm{ACO} O A C$
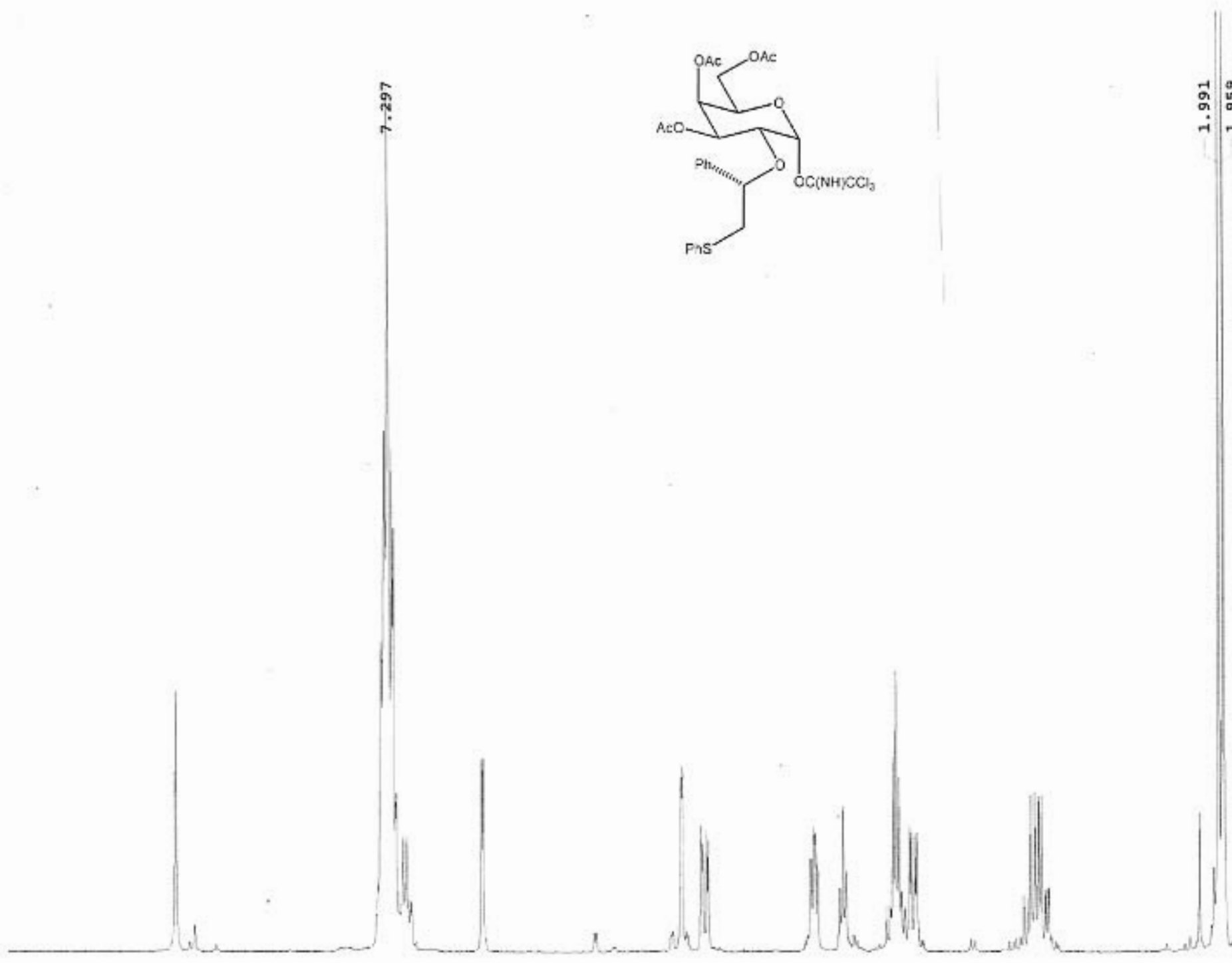

$$
{ }_{\mathrm{Ph}}^{\mathrm{O}} \mathrm{O}_{\mathrm{CCl}}
$$

虫

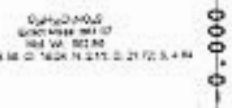

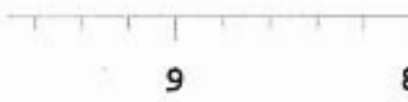

0.93
8

7

10.71

1.00

1.11
.06

5

3

2

1

$-0$

$1.08 \quad 1.20$

2.34

2.782 .63

2.99 


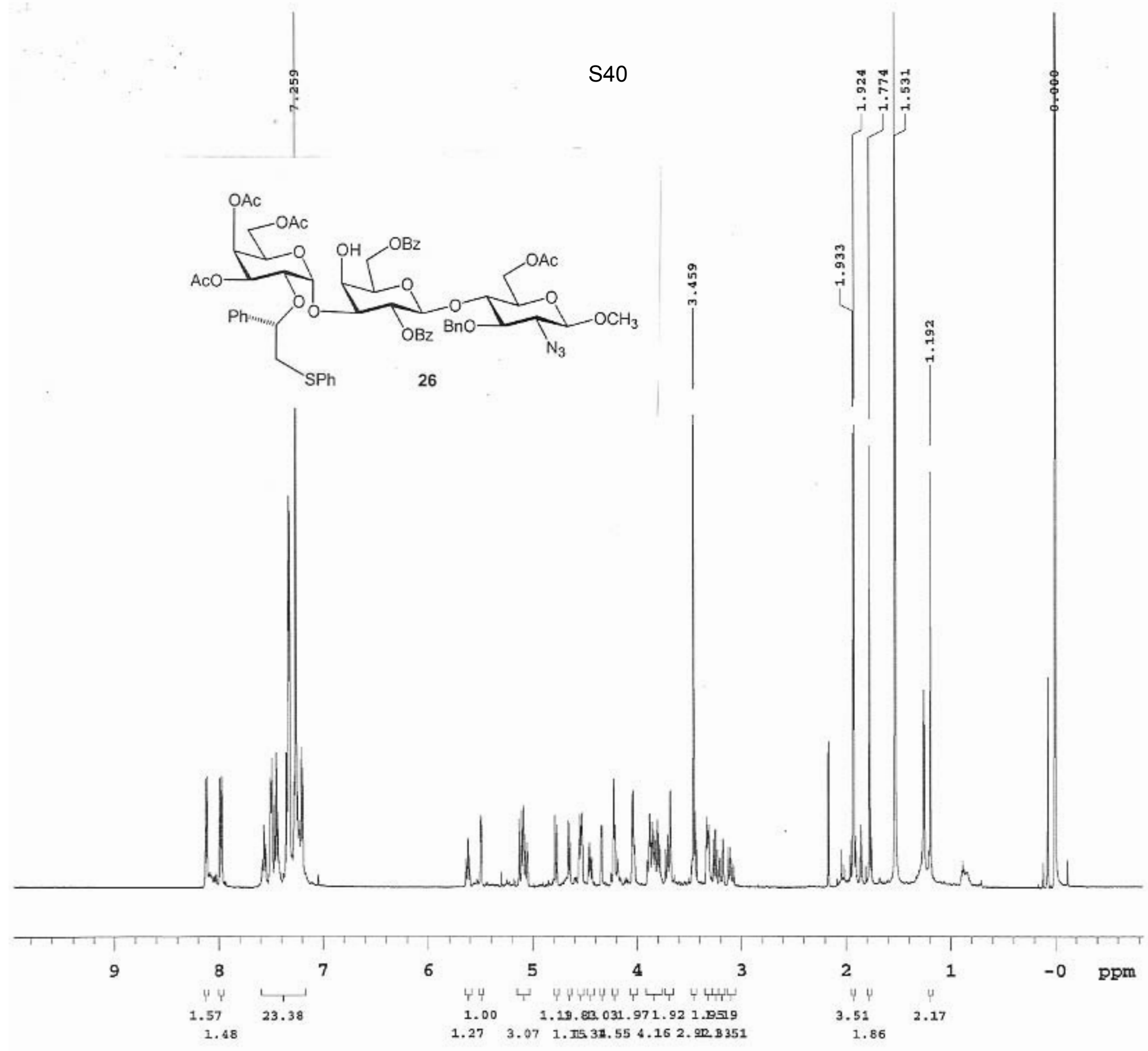




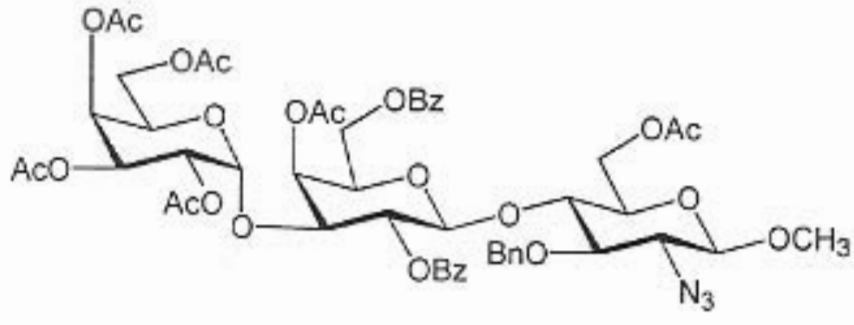

27

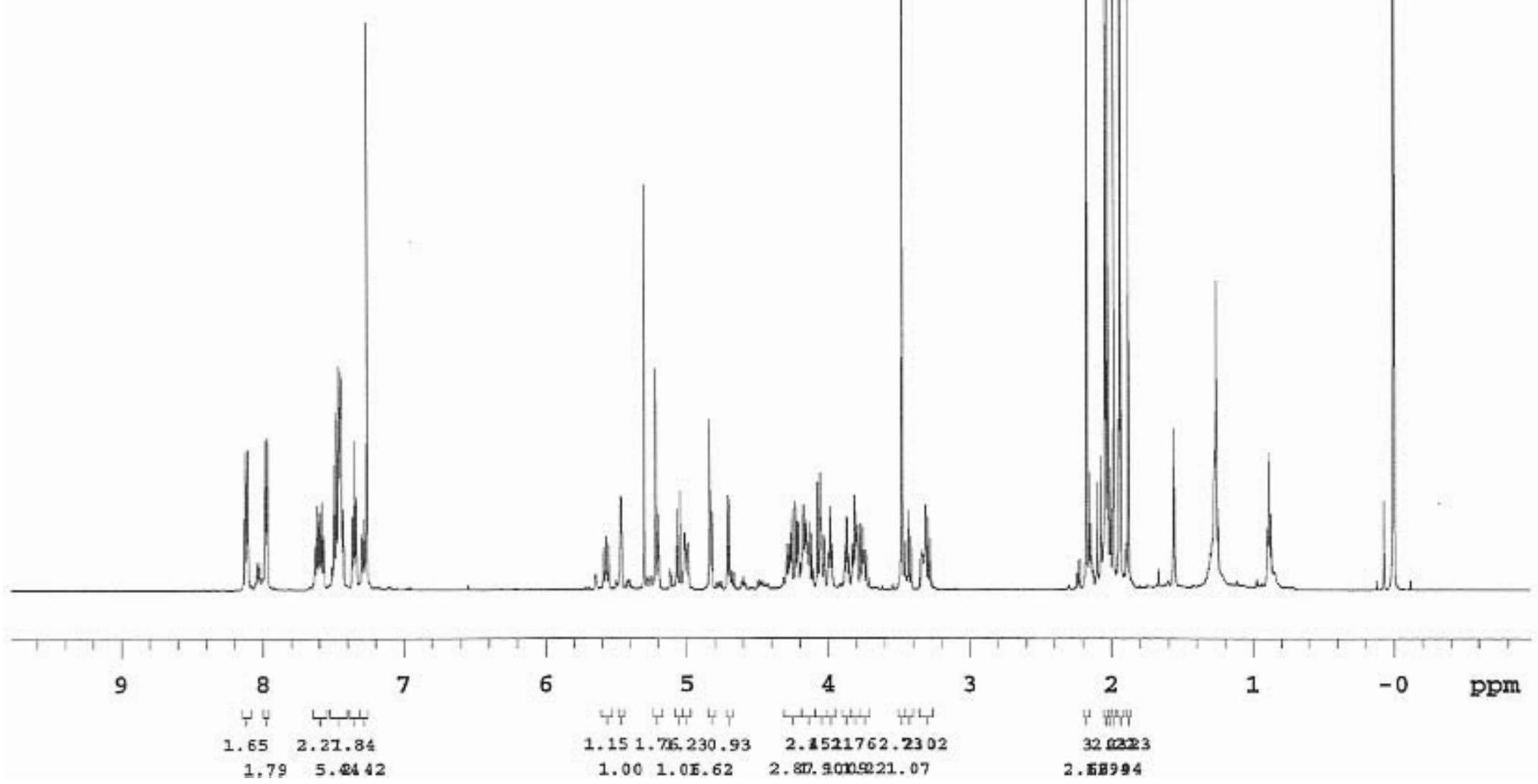


Archive directory: /export/home/kjhchem/vnmrsys/dat sample directory:

Pulse Sequence: : $2 \mathrm{pul}$

Solvent: $\operatorname{CDCl} 3$

Temp. $25.0 \mathrm{C} / 298.1 \mathrm{~K}$

Operator: kjhchem

Pile: Bn-donor

ONITYPlus-300 "nmr1"

Relas. delay $1.000 \mathrm{sec}$

Pulse 45.0 degrees

Acq. time $1.892 \mathrm{sec}$

width $8000.0 \mathrm{~Hz}$

8 repetitions

OBSERVE H1, $499.9407446 \mathrm{MHz}$

DATA PROCESSING

Line brodening $0.3 \mathrm{~Hz}$

PT size 32768

Total time 0 min, 29 sec

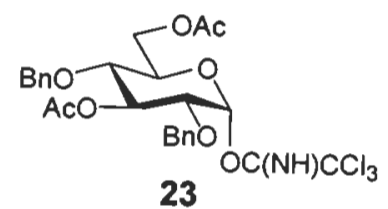

23

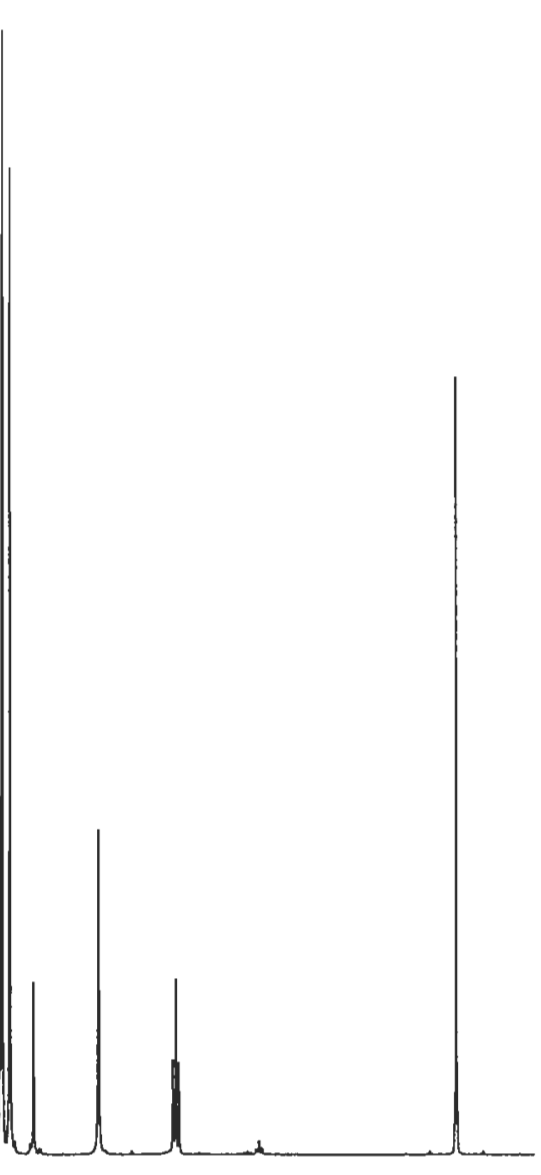

9

8

7

6

5

4

3

2

1

ppm 
Archive directory: /export/home/kjhchem/vnmrsys/data Sample directory:

Pulse Sequence: s2pul

Solvent: $\mathrm{CDCl} 3$

Temp. $25.0 \mathrm{C} / 298.1 \mathrm{~K}$

operator: kjhchem

File: kjh-597

UNITYP1us-300 "nmr1"

Relax. delay $1.000 \mathrm{Bec}$

Pulse 45.0 degrees

Acq. time $1.892 \mathrm{sec}$

Width 8000.0 Hz

16 repetitions

OBSERVE \#1, 499.9407510 MHz

DATA PROCE

Line broadening $0.3 \mathrm{~Hz}$

FT Bize 32768

rotal time 0 min, 52 sec
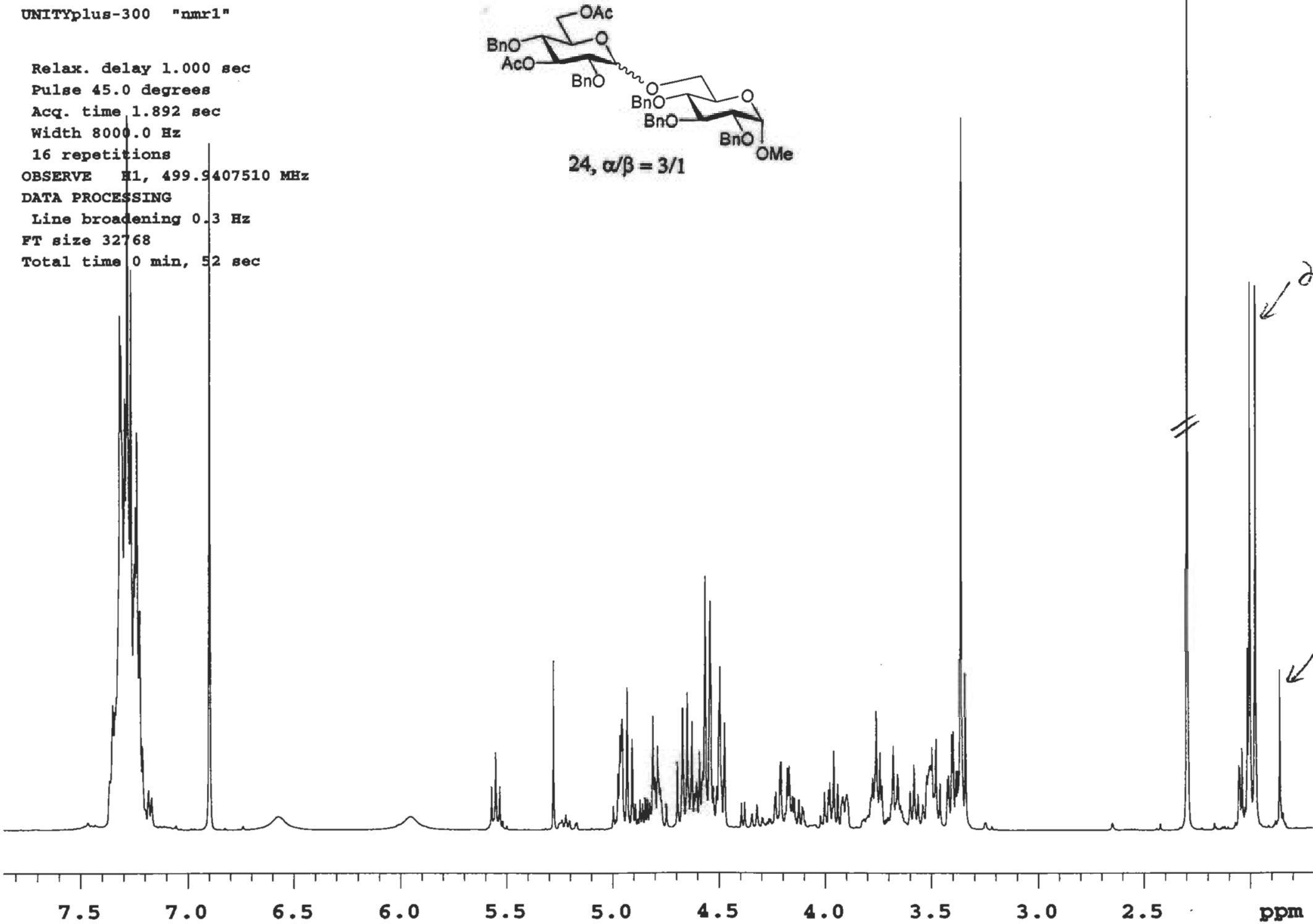


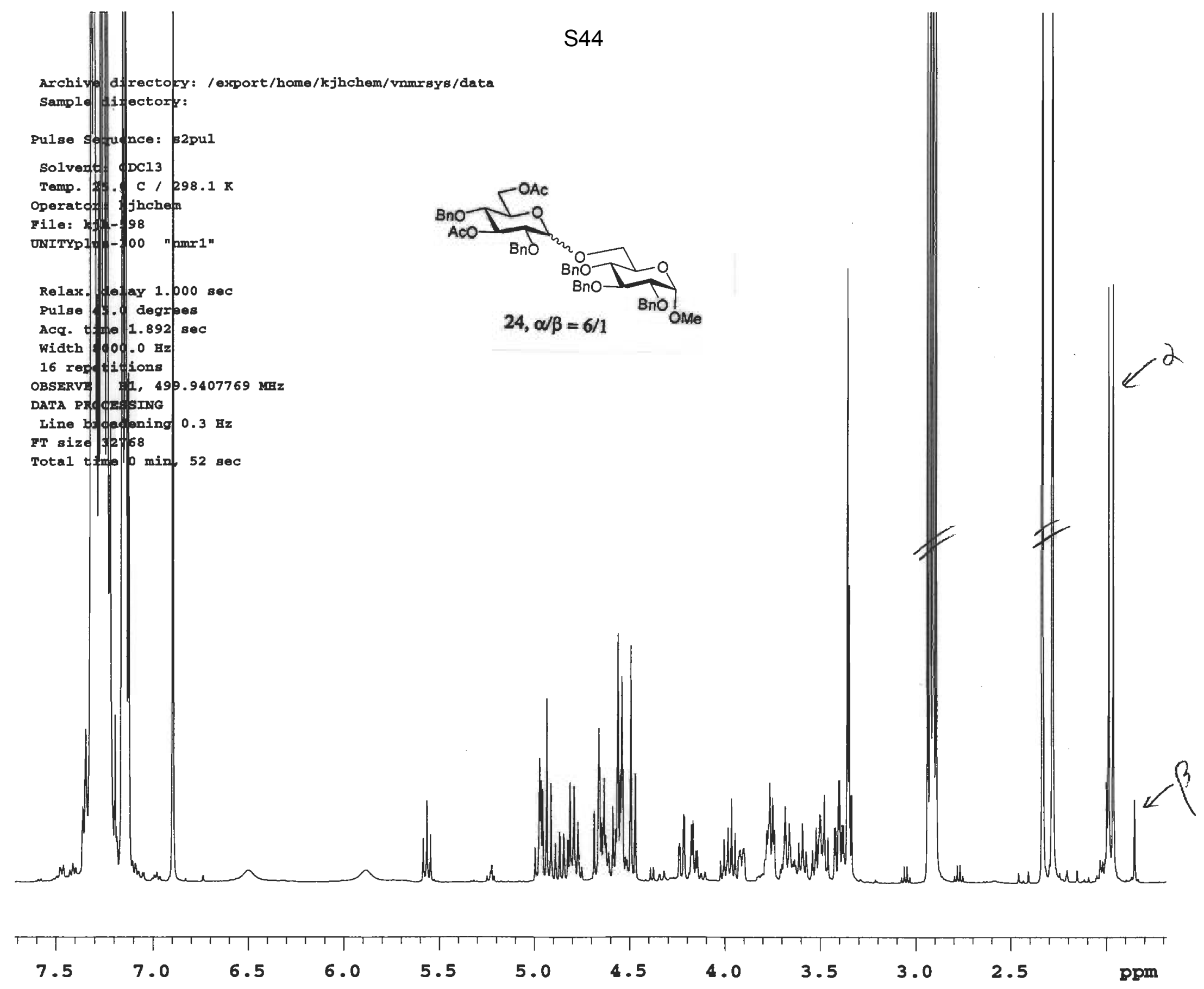




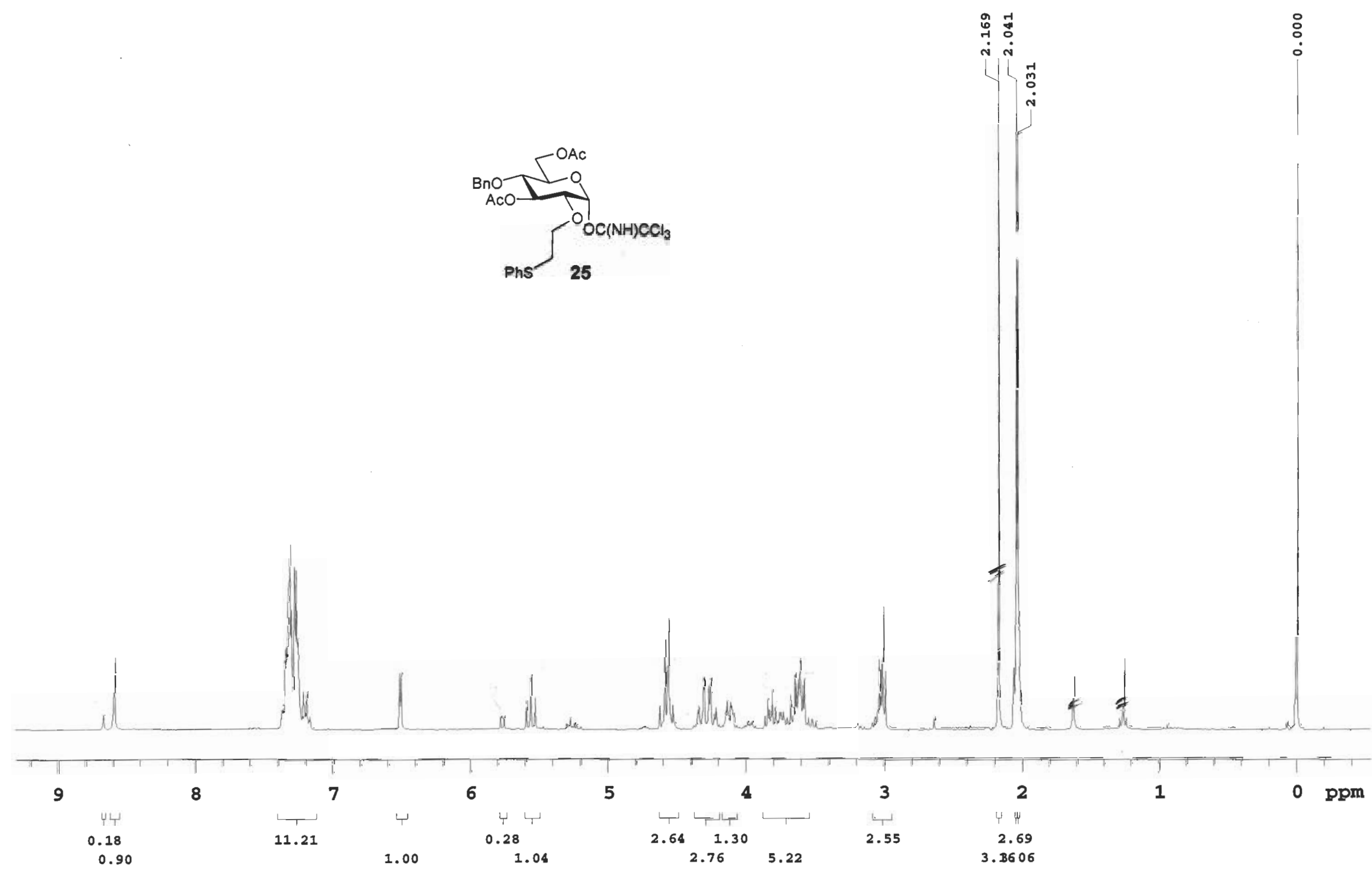


Archive directory: /export/home/kjhchem/unnrsys/data

Sample diractory:

\section{Pulse Sequeace: s2pul}

Solvent: $\mathrm{CDCl}_{3}$

Temp. 25.0 C / $298.1 \mathrm{~K}$

Operator: $\mathrm{kjhchem}$

File: kjh-595-2

UNITYPlus-300 "nmr1"

Relax. deley $1.000 \mathrm{sec}$

Pulse 45.0 degrees

Acq. time $1.892 \mathrm{sec}$

Width $8000.0 \mathrm{~Hz}$

16 repetitions

OBSERVE HI, $499.9407446 \mathrm{MHz}$

OBSENe

Line broadening $0.3 \mathrm{~Hz}$

FT size 32768

Total time 0 min, $52 \mathrm{sec}$

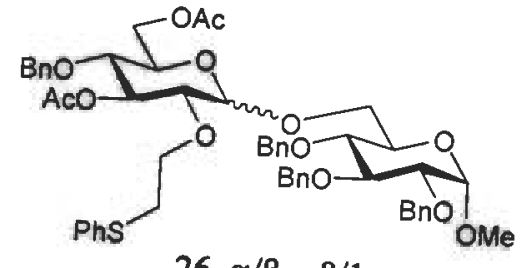

$26, \alpha / \beta=8 / 1$
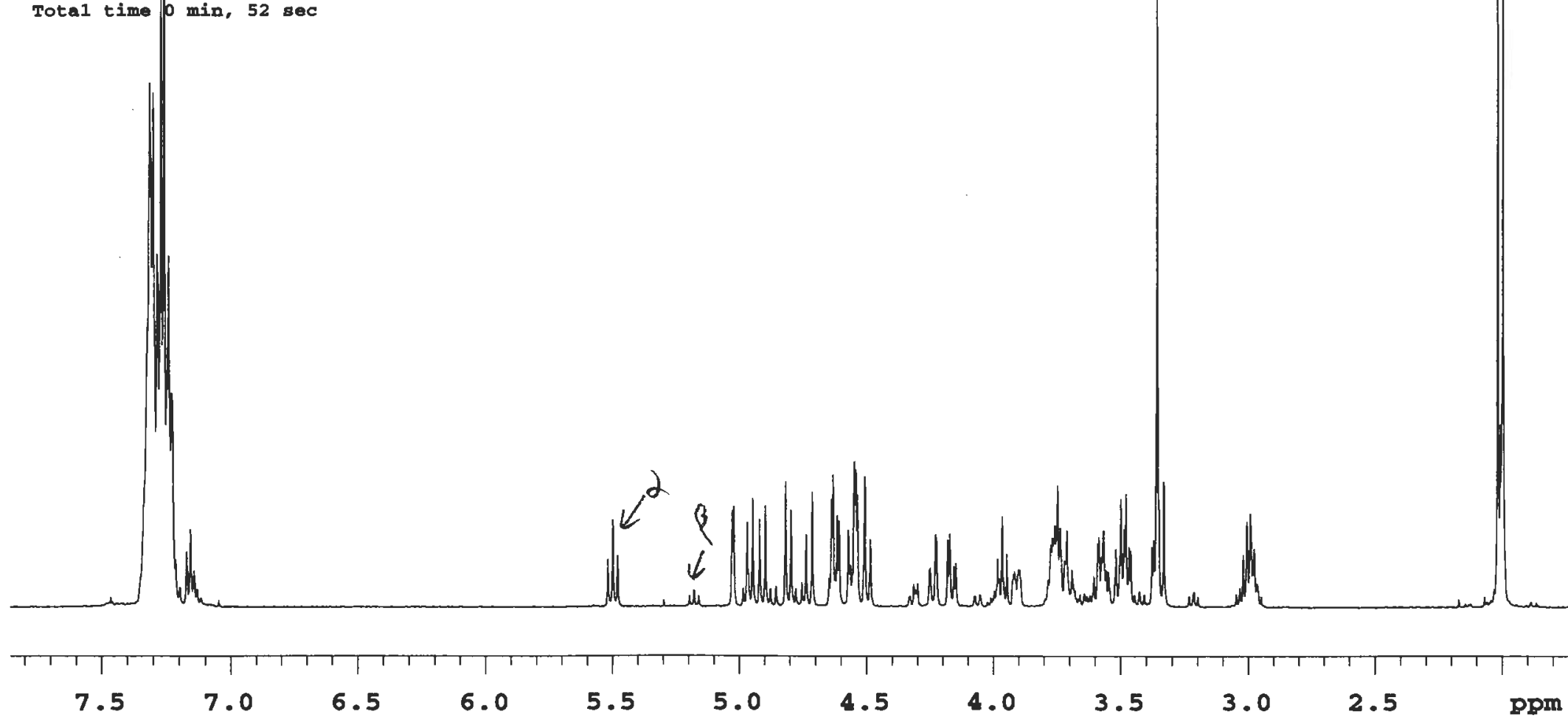
Archive directory: /export/home/hyang/vnmrsys/data

Sample directory: /export/home/hyang/vnmrsys/data/hyang_23Jun2004 File: PROTON

Pulse Sequence: s2pul

Solvent: $\mathrm{CDCl} 3$

Tenp. $25.0 \mathrm{C} / 298.1 \mathrm{~K}$

INOVA-500 "inOVa500"

Relax. delay $1.000 \mathrm{sec}$

Pulse 45.0 degrees

Acg. time $1.892 \mathrm{sec}$

Width $8000.0 \mathrm{~Hz}$

16 repetitions

OBSERVE H1, $499.9720030 \mathrm{MHz}$

DATA PROCESSTNG

FT size 32768

Total time 0 min, $46 \mathrm{sec}$
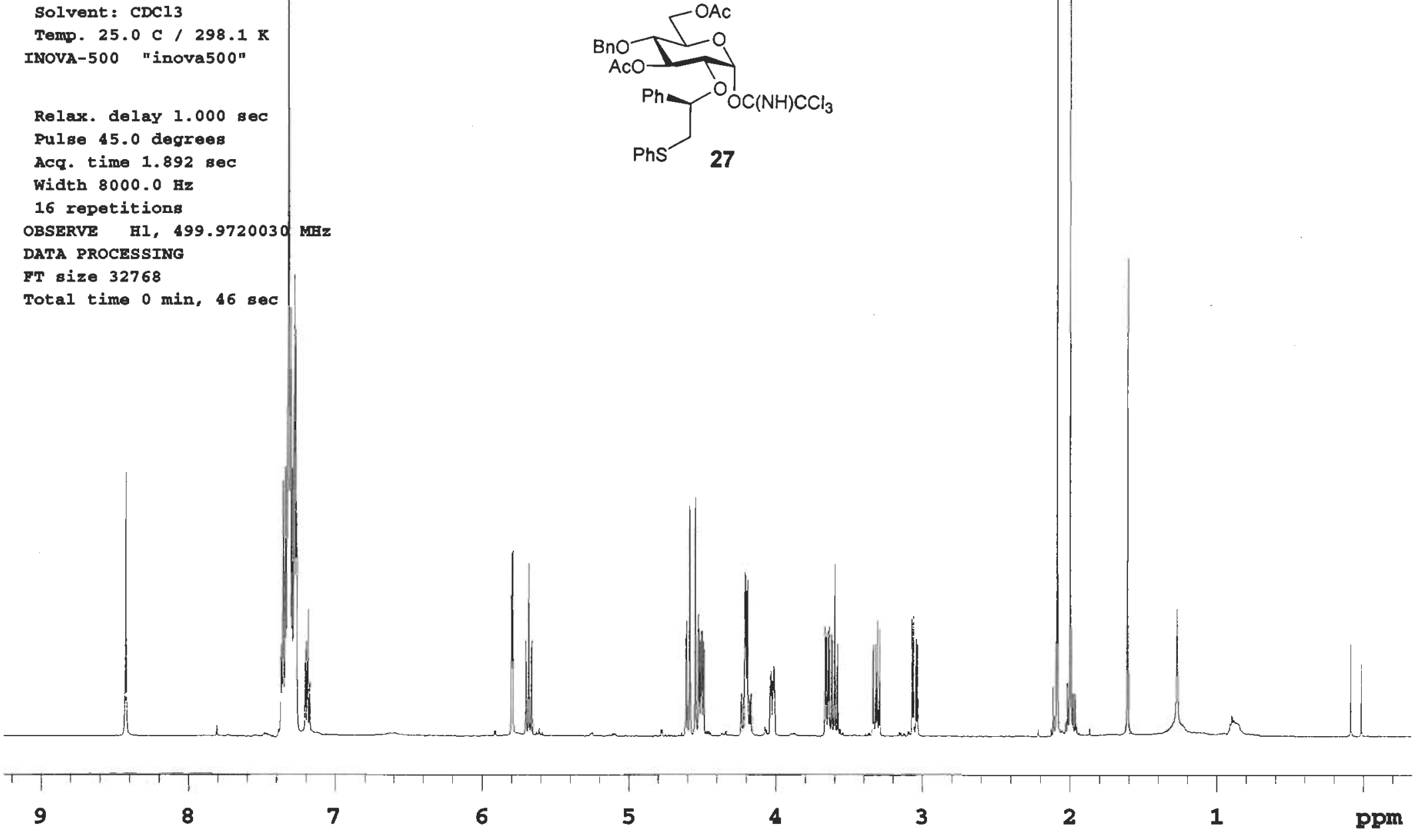
S48

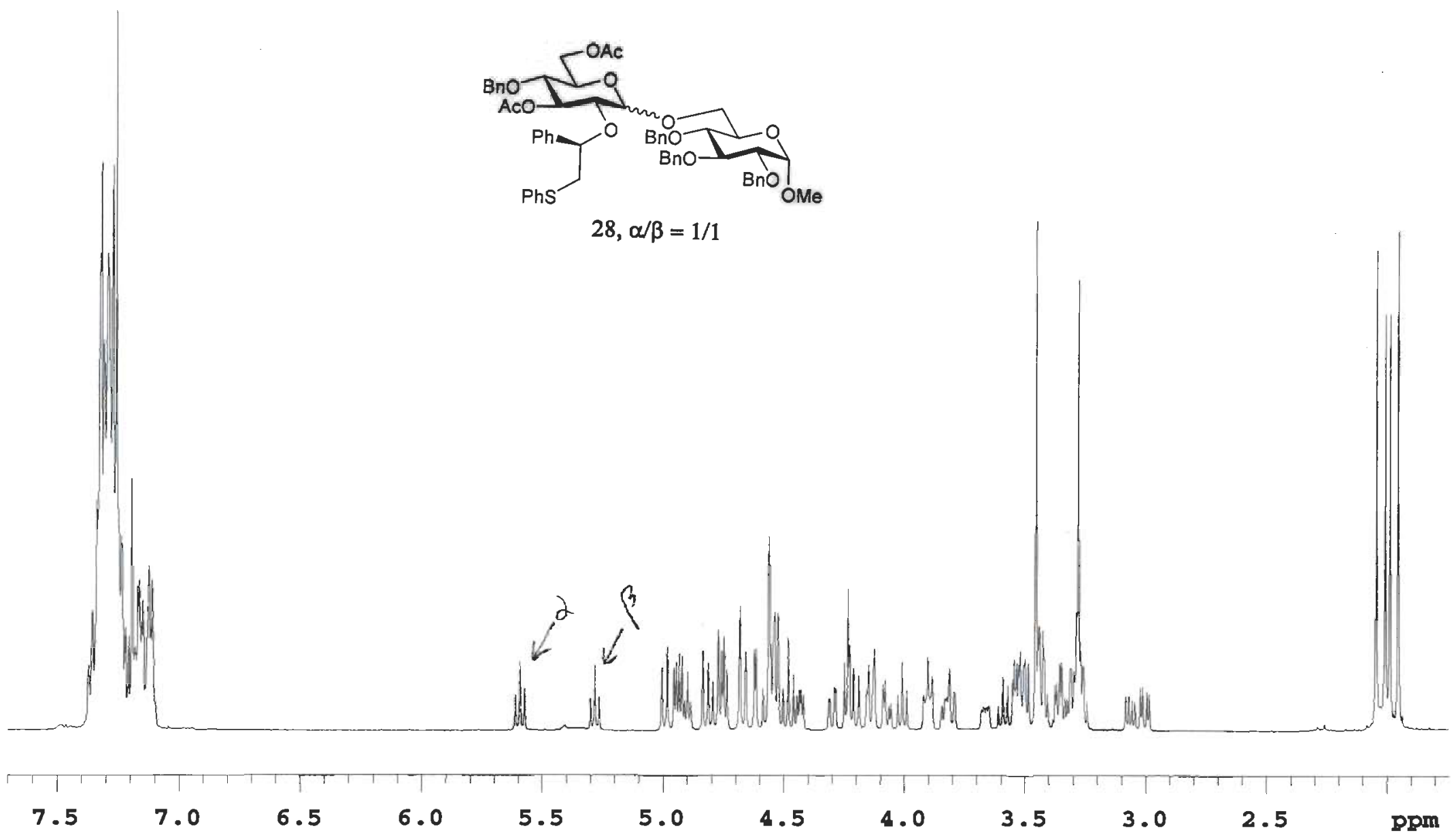

